Revised manuscript Submitted to "Pattern Recognition" on August 11, 2015

\title{
Fabric inspection based on the Elo rating method
}

\author{
Colin S. C. Tsang \\ Department of Mathematics \\ Hong Kong Baptist University, Kowloon Tong, Hong Kong \\ Email:11019700@life.hkbu.edu.hk \\ Henry Y. T. Ngan* \\ Department of Mathematics \\ Hong Kong Baptist University, Kowloon Tong, Hong Kong \\ Email: ytngan@hkbu.edu.hk \\ Phone: +852-3411-2531 \\ Grantham K. H. Pang \\ Industrial Automation Research Laboratory \\ Department of Electrical and Electronic Engineering \\ The University of Hong Kong, Pokfulam Road, Hong Kong \\ Email: gpang@eee.hku.hk
}

*Corresponding author 


\begin{abstract}
Automated visual inspection of patterned fabrics, rather than of plain and twill fabrics, has been increasingly focused on by our peers. The aim of this inspection is to detect, identify and locate any defects on a patterned fabric surface to maintain high quality control in manufacturing. This paper presents a novel Elo rating (ER) method to achieve defect detection in the spirit of sportsmanship, i.e., fair matches between partitions on an image. An image can be divided into partitions of standard size. With a start-up reference point, matches between various partitions are updated through an Elo point matrix. A partition with a light defect is regarded as a strong player who will always win, a defect-free partition is an average player with a tied result, and a partition with a dark defect is a weak player who will always lose. After finishing all matches, partitions with light defects accumulate high Elo points and partitions with dark defects accumulate low Elo points. Any partition with defects will be shown in the resultant thresholded image: a white resultant image corresponds to a light defect and a grey resultant image corresponds to a dark defect. The ER method was evaluated on databases of dot-patterned fabrics (110 defect-free and 120 defective images), star-patterned fabrics (30 defect-free and 26 defective images) and box-patterned fabrics ( 25 defect-free and 25 defective images). By comparing the resultant and ground-truth images, an overall detection success rate of $97.07 \%$ was achieved, which is comparable to the state-of-the-art methods.
\end{abstract}

Keywords: Elo rating, Sportsmanship, Match, Partition, Fabric inspection, Defect detection, Texture analysis, Patterned texture 


\section{INTRODUCTION}

Fabric is fundamental to many consumable products in daily life such as clothing, fashions, bags, bed coverings, nano-scale medical fabrics, and even air-conditioning ducts. Fabric inspection is a key component of quality control in textile manufacturing. Currently, most fabric inspection is conducted visually by human workers working at high cost, but it is not reliable due to human errors and eye fatigue. Automated visual inspection (AVI) of fabric applies computer vision techniques that offer not only an efficient, low-cost and accurate approach to replace the labour force but also expansion of inspection capabilities to cover a broader range of different fabric patterns, from the simplest to the most complicated. The aim of AVI is to detect and outline the shapes and locations of any defects on a fabric surface during or after weaving. There is much research on fabric inspection [1] of both simple and complicated patterned fabrics. This study focuses on fabric with complicated patterns (Fig. 1). A patterned fabric is composed of a basic fundamental unit, called the motif [2], which can be generated into the whole pattern by certain rules of symmetry. Based on these predefined rules, a patterned fabric can be classified into one of seventeen wallpaper groups [2].

Currently, AVI of fabric can be broadly classified into two main categories: motif- and non-motif-based. A number of methods have been developed for non-motif-based inspection. Most methods have been designed for the simplest patterns of the $\mathrm{p} 1$ group, plain and twill fabrics [1]. The five representative inspection approaches are statistical (e.g., regularity 


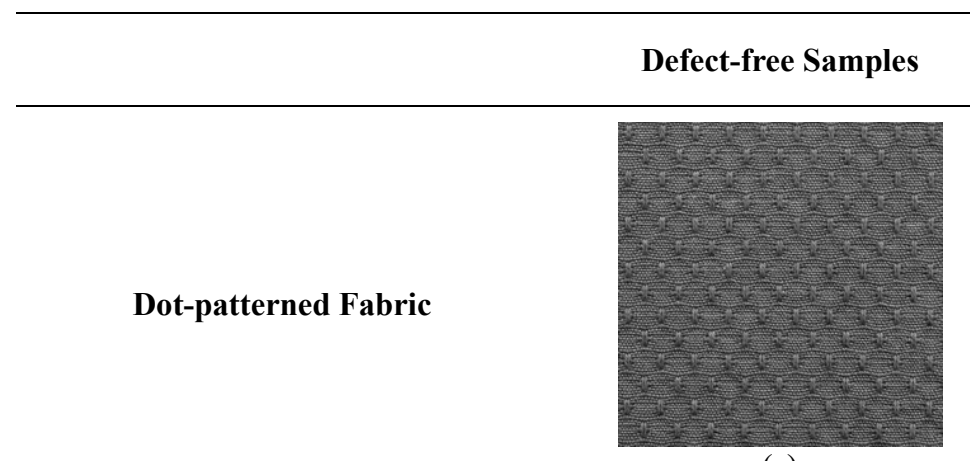

(a)

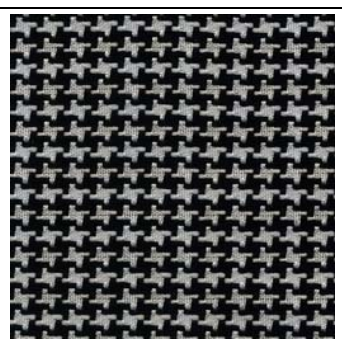

(c)
Defective Samples

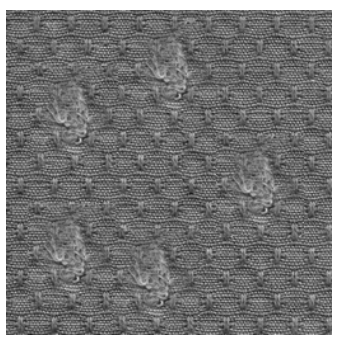

(b)

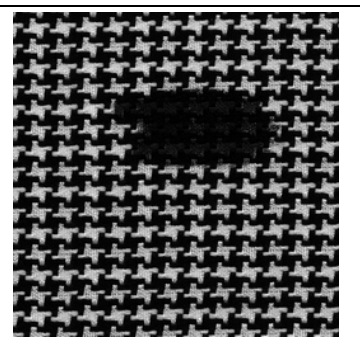

(d)

Fig. 1. Dot-patterned fabric images of (a) a defect-free sample and (b) a defective sample with light defects. Star-patterned fabric images of (c) a defect-free sample and (d) a defective sample with dark defects.

measure [3], fractal feature [4], morphological filter [5,6]), spectral (e.g., Fourier transforms [7-9], Gabor [10], wavelet [11-13]), model-based (e.g., Gaussian Markov random field [14], sparse dictionary reconstruction [15]), learning (e.g., neural network [16], support vector machine [4]) and structural (e.g., maximum frequency distance [17]). In contrast, only a few methods target other wallpaper groups, such as wavelet-pre-processed golden image subtraction (WGIS) [18], direct thresholding (DT) [18], co-occurrence matrix (CM) [19], Bollinger bands (BB) [20], regular bands (RB) [21] and image decomposition (ID) [22]. The method developed herein aims to inspect the patterned fabrics of the non-p1 fabric groups.

In this paper, a novel inspection method called the Elo rating (ER) method is proposed in which fabric inspection is treated as sporting matches between competing partitions (players). In other words, fabric inspection can be realised as sportsmanship during fairly 
played matches between competing partitions. The idea of the ER method originates from a logistic distribution-based statistical system called the Elo rating system, developed by Elo [23]. This system is used to measure a player's capability in many international chess matches, video matches [24] and even in many other international team sports including football, basketball, major league baseball, etc.

Fabric inspection is treated in the spirit of sportsmanship by the ER method, which provides a new perspective for the detection of fabric defects. The idea is that each $m \times n$ extracted image partition from an $M \times N$ testing image acts like a player. Some partitions are selected as players and each player is assigned a starting Elo point (W.L.O.G. $=1000$ as the starting number of base points). A player who wins a match gains some Elo points w.r.t. a formula suggested by Elo [23] based on a logistic distribution; otherwise the player loses some Elo points. In the long run, the Elo points accumulated are a fair indication of the player's performance even though some players do not encounter each other in the matches. In patterned fabric defect detection, image partitions of a patterned fabric image are considered the players and a match is regarded as the matrix operation between these partitions.

According to a score matrix of the ER method, a partition with light defective regions (Fig. 1(b)) will act like a strong player who tends to achieve a high score in the match and probably wins many matches. A partition with dark defective regions (Fig. 1(d)) will act like a weak player who tends to have a low score in the match and probably loses many matches. A partition that is defect-free will act like an ordinary player who tends to have an average score 
and ties many matches. Hence, a strong player with light defects should be able to gain Elo points by winning many matches, whereas a weak player with dark defects should lose Elo points by losing many matches. Therefore, an area of an image can be indicated as defective by tracking, partitions with relatively high or low Elo points. Match making in the ER method is designed as follows. For each partition (player), a certain number of other partitions (players) are randomly selected to have matches against it. The Elo points of a corresponding player will be updated after each match. In this paper, dot-, box-, and star-patterned fabrics comprising a total of 336 images (165 defect-free and 171 defective) are used.

This paper makes the following contributions to the literature.

1. A new application of the ER method is constructed by transforming its theoretical and physical properties for the purpose of patterned fabric inspection. Four key parameters in the ER method, including partition size, number of randomly located partitions, $w$-variable, and constant $K$, are carefully justified in the performance evaluation.

2. The ER method provides a binary classification of the nature of the defect in the final resultant image: white as a light defect, grey as a dark defect and black as defect-free.

3. From fabric databases with ground-truth images, the ER method achieves $96.89 \%$ accuracy for dot-patterned fabrics using 110 defect-free and 120 defective images, $98.82 \%$ accuracy for star-patterned fabrics using 30 defect-free and 26 defective images and $99.07 \%$ for box-patterned fabrics using 25 defect-free and 25 defective images. These results outperform those of the WGIS method and are comparable to those of the previously developed 
$\mathrm{BB}, \mathrm{RB}$ and ID methods in [22].

The remainder of this paper is organized as follows. In section 2, we survey the literature on the detection of patterned fabric defects. Section 3 outlines the ER method and its procedures. In section 4, we evaluate the performance of the ER method and compare it with the WGIS, BB, RB and ID methods. Lastly, section 5 concludes the paper.

\section{LITERATURE REVIEW ON DEFECT DETECTION IN PLAIN AND TWILL FABRICS}

The AVI techniques for fabrics in motif-based classification [25] can be divided into two main categories. Only a few methods have been developed for the patterned fabrics of the nonp1 wallpaper group. Research into the inspection of these patterned fabrics has been increasing in the last decade. The developed methods can be classified into four approaches: statistical, spectral, model-based and learning approaches.

The statistical approach includes gray relational analysis with CM features [19] on Jacquard fabric images to study correlations between the analysed factors of chosen features from a randomised factor sequence. This method reached $94 \%$ detection accuracy for 50 defective samples in [19]. The spectral approach includes many methods, such as WGIS [18], DT [18], wavelet-decomposition [26], template matching for discrepancy measure (TMPM) [27], BB [20], RB [21] and a Gabor filter [28]. The WGIS method [18] used a golden image to perform moving subtraction of each pixel along each row of every wavelet-pre-processed tested image. It generated $96.7 \%$ accuracy on 30 defect-free and 30 defective patterned images (pmm group). The DT method [18] applied a thresholding technique for defect detection on 
level 4 of Haar wavelet horizontal and vertical detailed sub-images in the same database for the WGIS method and generated $88.3 \%$ accuracy. Li and Di [26] developed a waveletdecomposition method [26] to improve the DT method by extracting Haar level 2 highfrequency coefficients as the vertical detailed sub-image for thresholding and applied a morphological filter to remove noise on a detected image. However, only one inspection on a knitted fabric sample was published. Stubl et al. [27] used a golden image-like approach to exploit a discrepancy measure as a fitness function to detect defects on patterned textures. They evaluated the TMPM method on the TILDA database (no details on the wallpaper group) and achieved 96.1\% correct detection. The BB and RB methods, designed by different combinations of moving average and standard deviation, used the regularity property of a patterned texture to carry out defect detection on dot-, box- and star-patterned fabrics (p2, pmm and $\mathrm{p} 4 \mathrm{~m}$ groups, respectively). The BB and RB methods obtained accuracy rates of $98.59 \%$ (167 defect-free and 171 defective images) and 99.4\% (80 defect-free and 86 defective images), respectively. Raheja et al. [28] proposed a Gabor filter method and a gray-level CM method that were then evaluated on 60 defect-free and defective samples (no details on the wallpaper group) from various fabrics with $98.33 \%$ and $95 \%$ correct detection, respectively.

The model-based approach includes a recent ID method [22] that decomposed a fabric image into structures of cartoon (defective objects) and texture (repeated patterns). The ID method resulted in detection accuracies ranging from $94.9 \%-99.6 \%$ for the dot-, box- and starpatterned fabrics. The ID method is carried out in a semi-supervised approach which is 
different to the WGIS, BB and RB methods which are performed in a supervised approach.

Lastly, in the learning approach, $\mathrm{Li}$ et al. [29] applied a spectral estimation technique to extract pattern features and fed them into a rough set classifier. They obtained $95.3 \%$ detection accuracy for a patterned fabric database (p4m group), in which 100 samples were used for training and 120 samples were used for testing.

In short, no previous method has viewed fabric inspection as a series of matches between any two partitions of the image. A novel use of the ER method for fabric defect detection is thus presented.

\section{THE ER METHOD}

\subsection{Definitions for the ER method}

Definition 1 (Score of a competition). For an image of size $m \times n$, say $G_{a}$, which has a match with another image of size $m \times n$, say $G_{b}$, the score s for $G_{a}$ is defined by

$\mathrm{s}=\frac{1}{m n} \sum_{i=1}^{m} \sum_{j=1}^{n}\left(G_{a_{i, j}}-G_{b_{i, j}}\right)$.

Definition 2 (Expected value of a win). Suppose the Elo points of images $G_{a}$ and $G_{b}$ with size $m \times n$ before a match are $E_{a}$ and $E_{b}$ respectively. The expected value of $G_{a}$ winning the match, according to logistic distribution, is defined as

$\operatorname{Exp}_{a}=\frac{1}{1+10^{\left(E_{b}-E_{a}\right) / w}}$

Without loss of generality, the starting Elo points of $E_{a}$ and $E_{b}$ are both set at 1000 . In (2), $\operatorname{Exp}_{a}$ and $\operatorname{Exp}_{b}$ are the respective probabilities of $G_{a}$ and $G_{b}$ winning the game. Hence, note the mathematical property below: 
$\operatorname{Exp}_{a}+\operatorname{Exp}_{b}=\frac{1}{1+10^{\left(E_{b}-E_{a}\right) / w}}+\frac{1}{1+10^{\left(E_{a}-E_{b}\right) / w}}=\frac{10^{E_{a} / w}}{10^{E_{a} / w}+10^{E_{b} / w}}+\frac{10^{E_{b} / w}}{10^{E_{b} / w}+10^{E_{a} / w}}=1$.

As suggested by Elo [23], $\mathrm{w}=400$, which is called the $w$ - variable of the ER system, is set arbitrarily. For example, the Elo points of any image are stabilized at the loser being $600=$ starting Elo point -400 or at the winner being $1400=$ starting Elo point + 400. If the number is large, the rating scale will be stretched out.

Definition 3 (Elo points update). After a match, the new Elo points of an image $G_{a}$ are defined by

$E^{\prime}{ }_{a}=E_{a}+K\left(X_{a}-\operatorname{Exp}_{a}\right)$

where $E_{a}$ is the original Elo points, $E^{\prime}{ }_{a}$ is the updated Elo points, $K$ is a constant $(\mathrm{K}=16$ is used here) and $X_{a}-\operatorname{Exp}_{a}$ should be in between -1 and 1:

$X_{a}=\left\{\begin{array}{l}1 \quad \text { if } G_{a} \text { wins the match } \\ 0.5 \quad \text { if } G_{a} \text { ties the match } \\ 0 \quad \text { if } G_{a} \text { loses the match }\end{array}\right.$

The constant $K$ in (4), regarded as the maximum Elo points an image can gain or lose in one match, can be set arbitrarily, but its scale would be excessive at a large value. If $K=16$ and $w=400$, an image of "high skill level to win matches" will gain around 10 Elo points per win, and vice versa. Similarly, an image's Elo points will be stabilized at $1400=$ starting Elo point +400 if the image continues to win matches, and vice versa.

The image in Definitions 1-3 above means the partition as described below.

\subsection{Procedures of the ER method}

The ER method involves three main phases: (A) acquisition of a score matrix, (B) the 
training stage and $(\mathrm{C})$ the testing stage.

\section{(A) Threshold acquisition from a score matrix}

Fig. 2 illustrates the procedure of threshold acquisition from a score matrix (see Algorithm 1

in the Appendix) presented in algorithmic steps.

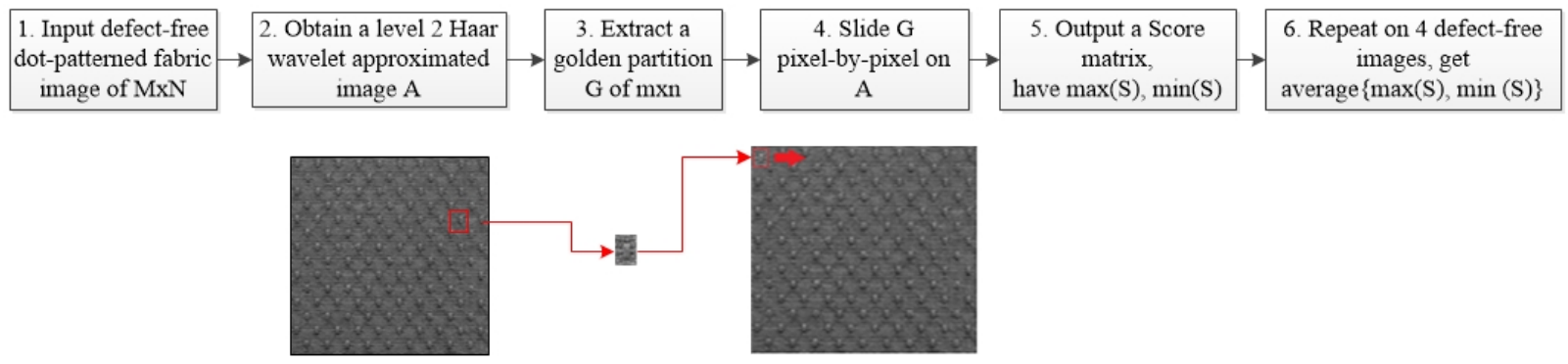

Fig. 2. Procedure for threshold acquisition from a score matrix.

1. Perform a level 2 Haar wavelet transform on defect-free images $R_{i}, \mathrm{i}=1, \ldots, \mathrm{k}$ to obtain corresponding approximated images $A_{i}, \mathrm{i}=1, \ldots, \mathrm{k}$ of size $M \times N$. A level 2 Haar approximated image is used because it reduces the computational time in subsequent stages (see the evaluation in Table 1). Level 2 strikes a balance between image size and computational time. The approximated image size of a level 3 Haar wavelet is $32 \times 32$, which would be too coarse for fabric inspection, and it is not considered.

Table 1

Computational requirements of different levels of wavelet extraction

\begin{tabular}{|c|c|c|c|c|}
\hline & & $\begin{array}{l}\text { Without } \\
\text { wavelet } \\
\end{array}$ & $\begin{array}{c}\text { Level } 1 \\
\text { Haar wavelet } \\
\end{array}$ & $\begin{array}{c}\text { Level } 2 \\
\text { Haar wavelet } \\
\end{array}$ \\
\hline & Image size & $256 \times 256$ & $128 \times 128$ & $64 \times 64$ \\
\hline \multirow{2}{*}{$\begin{array}{l}\text { Computational } \\
\text { time }\end{array}$} & Training stage & $273.54 \mathrm{~s}$ & $52.96 \mathrm{~s}$ & $12.38 \mathrm{~s}$ \\
\hline & Testing stage & $50.51 \mathrm{~s}$ & $9.47 \mathrm{~s}$ & $1.97 \mathrm{~s}$ \\
\hline
\end{tabular}


2. For each $M \times N$ level 2 Haar wavelet transformed approximated image, extract a golden partition $G_{k}$ of size $m \times n$, where $m \times n$ is roughly half the dimensions of the length and width of a motif (each patterned texture is generated by a motif [2]).

3. Slide $G_{k}$ on each pixel along each row $\mathrm{p}$ on $A_{k}$. For each move of the sliding process, record the corresponding score $s_{x, y}$ in each match, where $\mathrm{x} \in \mathrm{M}, \mathrm{y} \in \mathrm{N}$.

4. Output a score matrix $S=\left\{s_{x, y}\right\}^{k}$ of size $(M-m+1) \times(N-n+1)$ and extract $\max \left(S_{k}\right)$ and $\min \left(S_{k}\right)$.

5. Repeat steps 1-4 for four defect-free sample images (as $\mathrm{k}=5$ in total). Thus we can define Threshold $_{\text {win }}=\overline{\max \left(S_{k}\right)}=\overline{S_{k_{\max }}} \quad$ and $\quad$ Threshold $_{\text {loss }}=\overline{\min \left(S_{k}\right)}=\overline{S_{k_{\min }}}$, where the bar sign means to obtain either the average value of all $\max \left(S_{k}\right)$ or that of all $\min \left(S_{k}\right)$

6. Determine a win/tie/loss for any given match, with the interval between Threshold $_{\text {win }}$

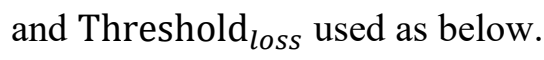

If Score $>$ Threshold $_{\text {win }}$, the match is defined as a win.

If Threshold loss $_{<\text {Score }}<$ Threshold $_{\text {win }}$, the match is defined as a tie. If Score $<$ Threshold $_{\text {loss }}$, the match is defined as a loss.

\section{(B) Training stage}

1. Perform level 2 Haar wavelet transform on a defect-free image to obtain approximated images $A_{k}$ of size $M \times N$ (see Algorithm 2 in the Appendix for details). 
2. Divide the approximated images into many partitions. Extract $(M-m+1) \times(N-n+$ 1) partitions for each of size $m \times n$. For example, the approximated image $A_{k}$ of a dotpatterned fabric image is of size $64 \times 64$ and its motif is roughly of size $7 \times 4$. Therefore, $(64-7+1) \times(64-4+1)=58 \times 61=3538$ partitions of size $7 \times 4$ are extracted for each sample. Name these partition $P_{1.1}, P_{1.2}, \ldots, P_{58.61}$ according to their location.

3. Assign each partition a starting Elo points value (1000 is assigned here). Hence, a reference matrix of Elo points $E=(1000)_{58 \times 61}$ is obtained for future update.

4. Perform a random locating process to have $r$ pairs of $(\mathrm{x}, \mathrm{y})$-coordinates. For $r$ randomly located partitions, say $\left(P_{x_{h_{i}}, y_{k_{i}}}\right)_{i=1,2, \ldots, r}$ (i.e., for each $\mathrm{x}$-coordinate $\left[x_{1}, x_{2}, \ldots, x_{61}\right]$ and ycoordinate $\left[y_{1}, y_{2}, \ldots, y_{58}\right]$, we randomly pick $r$ locations. Thus we have $\left[x_{h_{1}}, x_{h_{2}}, \ldots, x_{h_{r}}\right]$ and $\left.\left[y_{k_{1}}, y_{k_{2}}, \ldots, y_{k_{r}}\right]\right)$

5. Starting from $P_{1.1}, P_{1.1}$ will have $R$ matches with $\left(P_{x_{h_{i}}, y_{k_{i}}}\right)$, with the result of each match (win/tie/loss) decided by step 6 of (A). After each match, the corresponding location on the Elo point matrix $E_{k}$ will be updated by Definition 3 (Elo point update). Then obtain a new set of random located partitions $\left(P_{x_{h_{i}}, y_{k_{i}}}\right)$ by repeating step 4.

6. Keep sliding on each pixel on each row $x$, so we can repeat step 5 from $P_{1,1}$ to $P_{58,61}$

7. After step 6 has finished, an updated Elo point matrix $E$ is obtained:

$\left(\begin{array}{ccccc}E_{1,1} & E_{1,2} & \ldots & \ldots \\ \vdots & \vdots & & \ddots & \vdots \\ \ldots & \ldots & \ldots & E_{58,61}\end{array}\right)$.

Extract $\max (E)$ and $\min (E)$. 
8. Repeat steps 1-7 for five defect-free sample images. Thus, it has Threshold $_{\text {light }}=\overline{\max \left(E_{k}\right)}$ and Threshold dark $=\overline{\min \left(E_{k}\right)}$.

9. Update the Elo point matrix $E_{k}$ of a test image. If $E_{i, j}>$ Threshold ${ }_{\text {light }}$, the partition $P_{i, j}$ is defined as light defective (shown as white in the final image). If Threshold dark $<$ $E_{i, j}<$ Threshold $_{\text {light }}$, the partition $P_{i, j}$ is defined as defect-free. If $E_{i, j}<$ Threshold $d_{\text {dark }}$, the partition $P_{i, j}$ is defined as dark defective (shown as grey in the final image).

\section{(C) Testing Stage}

1. Carry out steps 1-6 of the training stage of Elo points to obtain an updated Elo point matrix $E$ of a defective image.

2. Perform defect detection according to step 9 of the training stage.

3. Output a three-colour (black-grey-white) resultant image $U$ :

$\left(\begin{array}{ccccc}U_{1,1} & U_{1,2} & \ldots & \ldots \\ \vdots & \vdots & & \ddots & \vdots \\ \ldots & \ldots & \ldots & U_{58,61}\end{array}\right)$

If $P_{i, j}$ is light defective, $U_{i, j}=1$ (shown as white in the final image).

If $P_{i, j}$ is dark defective, $U_{i, j}=0.5$ (shown as grey in the final image).

If $P_{i, j}$ is defect-free, $U_{i, j}=0$ (shown as black in the final image).

\subsection{Application of a median filter on $U$ to remove noise}

A fabric sample with a light defect will be shown as a white resultant image and a fabric sample with dark defect will be shown as a grey resultant image for visualisation purposes. Fig. 
3 shows examples of final resultant images from the ER method with their corresponding mesh diagrams. A defective sample of Knots $(\mathrm{k} 3)$ in the second column is considered a light defect and shows a white resultant image, whereas the defective samples of Thin Bar (t1), Thick Bar (tt1), Netting Multiple (n1), Broken End (b1) and Hole (h1) are regarded as dark defects and are shown as grey resultant images. 


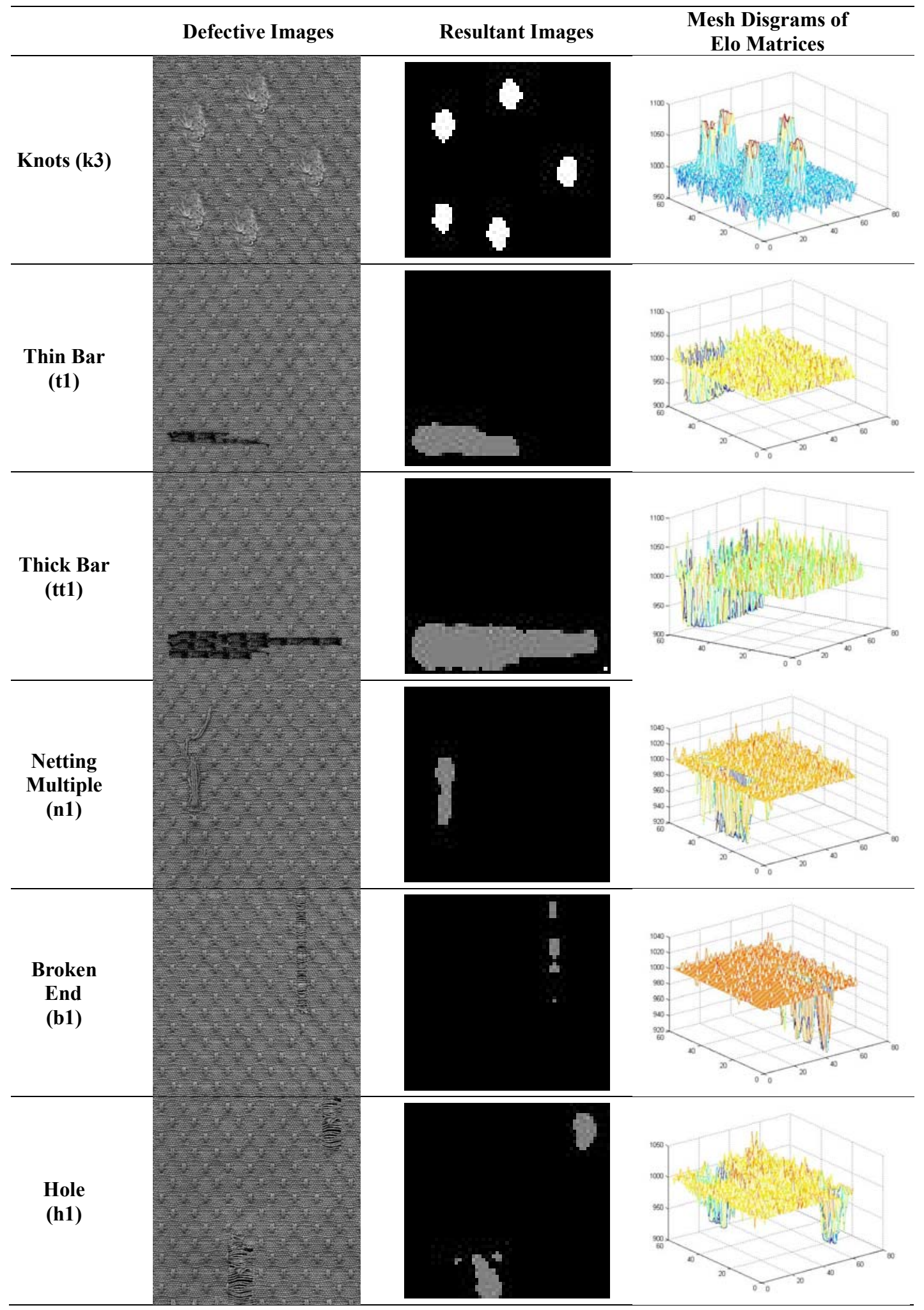

Fig. 3. $\left(1^{\text {st }}\right.$ column$)$ Defective sample names; $\left(2^{\text {nd }}\right.$ column $)$ Defective images; $\left(3^{\text {rd }}\right.$ column $)$ Detection results; ( $4^{\text {th }}$ column) Mesh diagrams of the Elo matrices. 


\section{PERFORMANCE EVALUATION}

In total, 336 images of size $256 \times 256$ in 24-bit depth from the dot-, box- and star-

patterned fabric databases are used for evaluation. The dot-patterned fabric database contains 110 defect-free and 120 defective images, the star-patterned database contains 30 defect-free and 26 defective images, and the box-patterned fabric database contains 25 defect-free and 25 defective images. All defective images have corresponding binary ground-truth images that illustrate the defective regions as white (value 1) and the defect-free regions as black (value 0 ). Performance evaluation is carried out in two progressive steps. In the first step, the number of white pixels in the final resultant image is obtained. In the second step, a number of measurement metrics are obtained, namely true positive (TP), false positive (FP), true negative (TN), false negative (FN), detection success rate (DSR), true positive rate (TPR), false positive rate (FPR), positive predictive value (PPV) and negative predictive value (NPV). The details of these metrics are provided in [22].

In the first step, the number of white/grey pixels extracted by the thresholding in the ER method is obtained. However, this cannot fully reveal the detection performance because the white/grey pixels can actually be false alarms. Therefore, the second step provides a detailed analysis. The TPR and FPR are useful for an analysis of the TPR-FPR graph. The PPV can also be treated as a measure of precision on the fraction of the TP cases among the number of positive calls in inspection. These metrics assist us in verifying how the ER method performs with various patterns. We use a desktop computer with an AMD Athlon ${ }^{\mathrm{TM}}$ X4 740 Quad Core 
320-GHz processor and $8.00 \mathrm{~GB}$ of memory. The programming language is MATLAB 7.0.

The initial parameters of the ER method a partition size of $7 \times 4$ and 16 randomly located partitions is 16 (which is selected as a reasonable choice due to an analysis of computational requirement of different number of randomly selected partitions in Table 2).

TABLE 2.

COMPUTATIONAL REQUIREMENT OF DIFFERENT NUMBER OF RANDOMLY SELECTED PARTITIONS

\begin{tabular}{|c|c|c|c|c|c|c|}
\hline $\begin{array}{l}\text { Number of } \\
\text { Randomly } \\
\text { Selected } \\
\text { Partitions }\end{array}$ & & 9 & 16 & 25 & 36 & 3538 \\
\hline \multirow{2}{*}{$\begin{array}{l}\text { Computational } \\
\text { Time (seconds) }\end{array}$} & Training Stage & $7.05 \mathrm{~s}$ & $12.38 \mathrm{~s}$ & $16.95 \mathrm{~s}$ & $25.46 \mathrm{~s}$ & $2199.09 \mathrm{~s}$ \\
\hline & Testing Stage & $1.20 \mathrm{~s}$ & $1.97 \mathrm{~s}$ & $3.07 \mathrm{~s}$ & $4.40 \mathrm{~s}$ & $460.30 \mathrm{~s}$ \\
\hline
\end{tabular}

A partition size $=7 \times 4$

\subsection{Tuning the parameters of the ER method}

In this section, four parameters are further tuned for the ER method: a) the partition size $m \times n, b)$ the number of randomly located partitions $r$, c) $w$ - variable in (2) and d) constant value $K$ in (4).

\section{a) Effect of the partition size $m \times n$}


Fig. 4 illustrates the DSR, TPR and FPR plots of the effect of the partition size, $m \times n$, on the dot-patterned (Fig. 4(a)), star-patterned (Fig. 4(b)) and box-patterned (Fig. 4(c) fabrics. The star- and box-patterned fabrics have undergone the same evaluation processes with the partition sizes of $3 \times 2,7 \times 4,10 \times 6,13 \times 8$ and $16 \times 10$. Fig. 4(a) and (b) clearly indicates that the $7 \times 4$ size offers the best TPR among the five choices (about $60 \%$ in the dot- and $20 \%$ in the star-patterned fabrics), whereas the TPR and FPR are quite stable at each size. In Fig. 4(c), $10 \times 6$ provides the best TPR (about 20\%) for the box-patterned fabric.

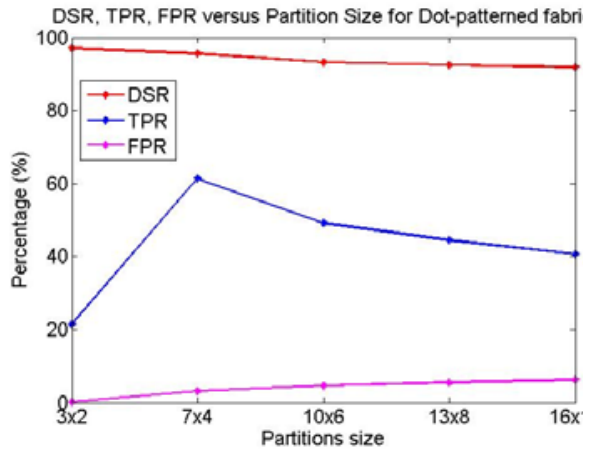

(a) dot-patterned

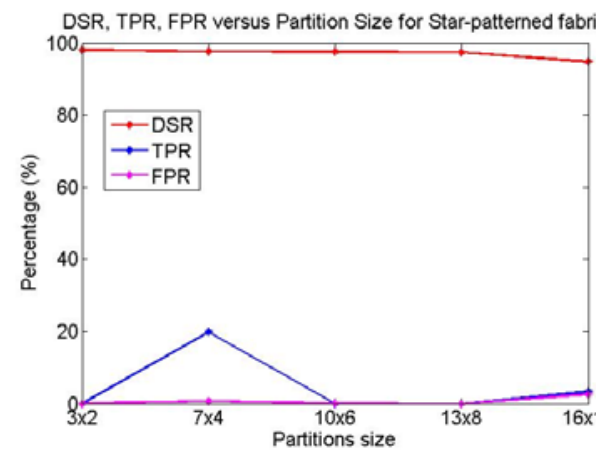

(b) star-patterned

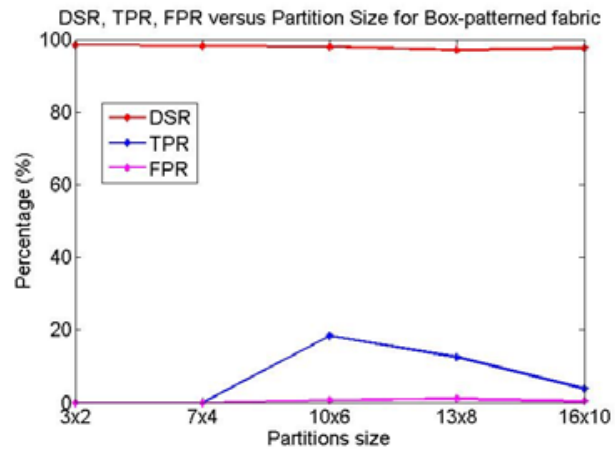

(c) box-patterned

Fig. 4. DSR, TPR and FPR versus number of partitions for the (a) dot-patterned, (b) starpatterned and (c) box-patterned fabrics. 


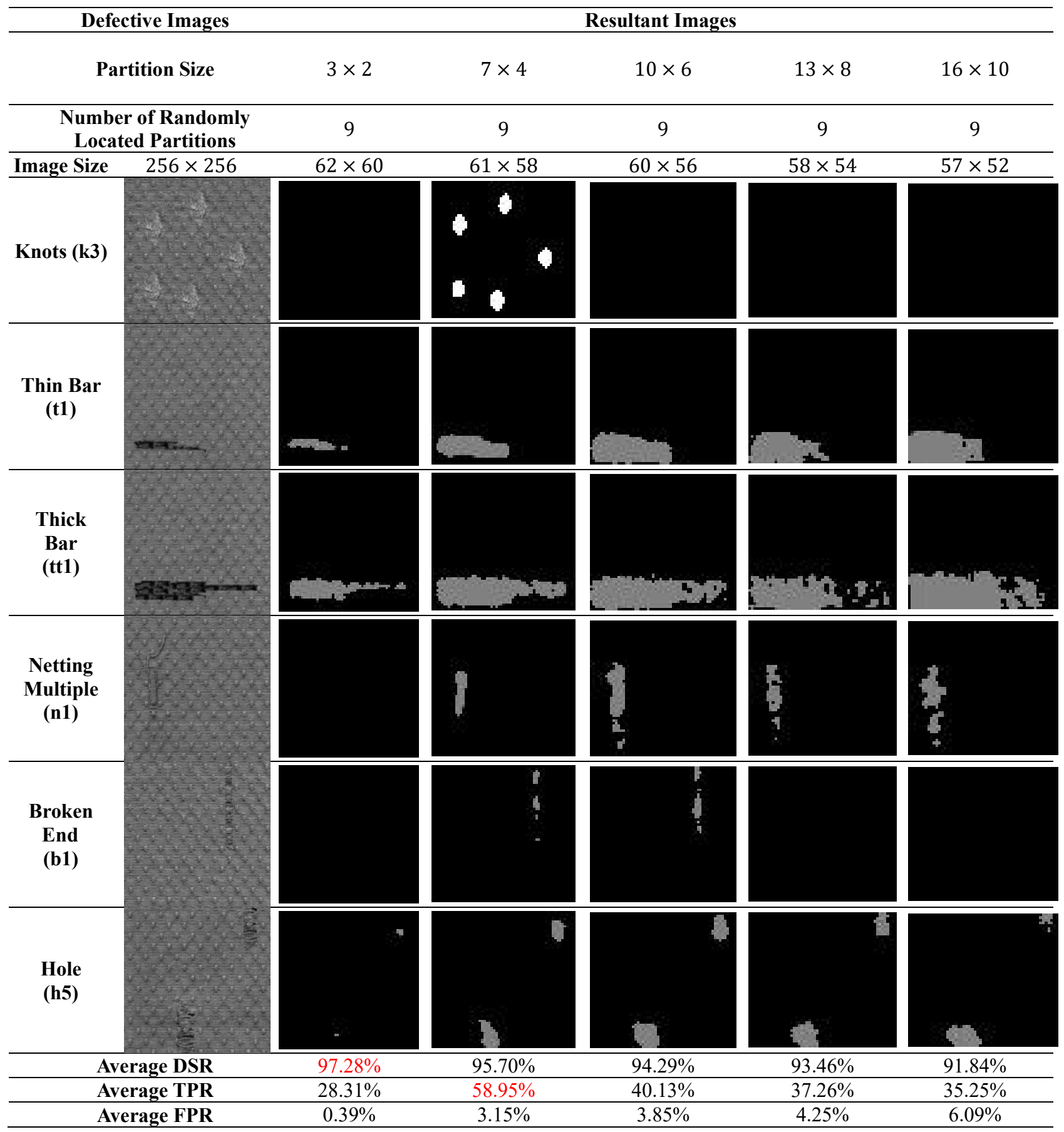

Fig. 5. Effect on the various partition sizes.

With respect to the different defective samples of the dot-patterned fabric, Knots, Thin

Bar, Thick Bar, Netting Multiple, Broken End and Hole, Fig. 5 depicts selected results of the

various partition sizes of a golden partition $G_{k}$ of size $m \times n: 3 \times 2,7 \times 4,10 \times 6,13 \times 8$,

$16 \times 10$ and $19 \times 12$. All of the procedures of the ER method remain the same. Although the 
size $3 \times 2$ generates the highest average DSR of $97.28 \%$, it actually misses many true defective regions. In contrast, the metric TPR reveals the performance more accurately: the size $7 \times 4$ provides an average TPR of $58.95 \%$, which is the highest among the three options.

\section{b) Effect of the number of randomly located partitions $r$}

For the second parameter, the number of randomly located partitions $r$ is tested from 5 to 60. Fig. 6 depicts the plots of DSR, TPR and FPR for this parameter. For the sake of computational efficiency, $r$ should be as small as possible for DSR, TPR and FPR to maintain reasonable good rates. In Fig. 6(a), TPR is $60.63 \%$ at $r_{0}=10$ and $64.94 \%$ at $r=15$ and continues to increase thereafter; however the computational demand also rises. Therefore, $r=$ 15 is an optimal choice for the dot-patterned fabric. Fig. 6(b) shows $r=40$ to be an optimal choice when the TPR reaches $31.07 \%$ and becomes stable for the star-patterned fabric. Fig.

6(c) shows that $r=15$ is an optimal choice for the box-patterned fabric because all TPRs

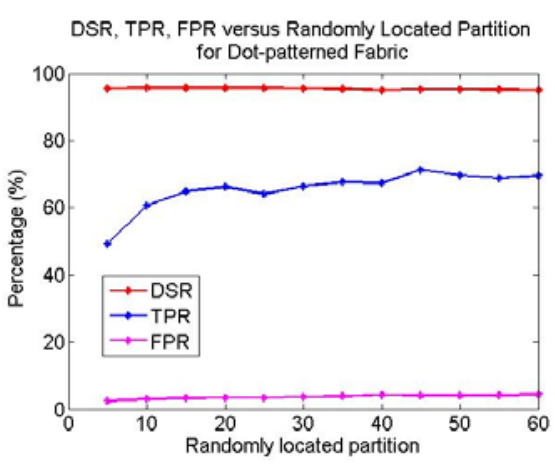

(a) dot-patterned

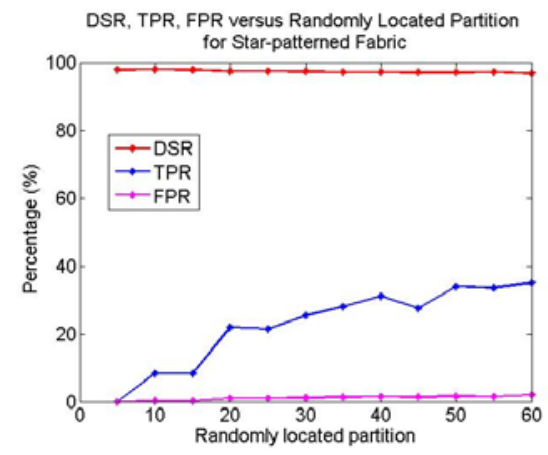

(b) star-patterned

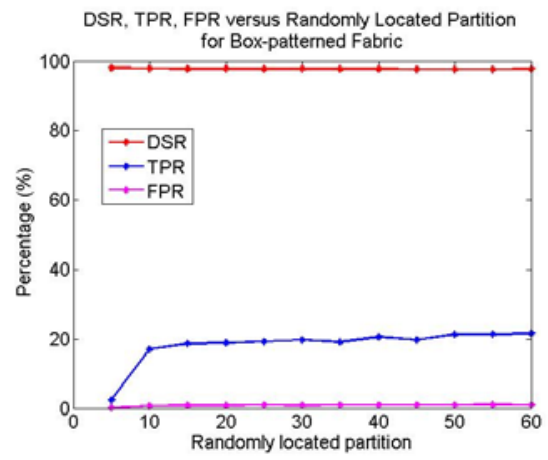

(c) box-patterned

Fig. 6. DSR, TPR and FPR versus number of randomly located partitions for the (a) dotpatterned, (b) star-patterned and (c) box-patterned fabrics. 
begin to be stable around $18 \%$ thereafter. For all $r$ values, the DSRs and FPRs in Figs.

$6(a),(b),(c)$ for all three patterned fabrics are all very stable at the levels of greater than $95 \%$

\begin{tabular}{|c|c|c|c|c|}
\hline \multirow{2}{*}{\multicolumn{2}{|c|}{$\begin{array}{c}\text { Defective images } \\
\text { Partition size }\end{array}$}} & \multicolumn{3}{|c|}{ Resultant images } \\
\hline & & $7 \times 4$ & $7 \times 4$ & $7 \times 4$ \\
\hline \multicolumn{2}{|c|}{$\begin{array}{l}\text { Number of randomly } \\
\text { located partitions }\end{array}$} & 9 & 16 & 25 \\
\hline Image Size & $256 \times 256$ & $61 \times 58$ & $61 \times 58$ & $61 \times 58$ \\
\hline Knots (k3) & & & & \\
\hline $\begin{array}{l}\text { Thin Bar } \\
\text { (t1) }\end{array}$ & & & & \\
\hline $\begin{array}{l}\text { Thick } \\
\text { Bar } \\
\text { (tt1) }\end{array}$ & & & & : \\
\hline $\begin{array}{l}\text { Netting } \\
\text { Multiple } \\
\text { (n1) }\end{array}$ & & & & \\
\hline $\begin{array}{l}\text { Broken End } \\
\text { (b1) }\end{array}$ & & 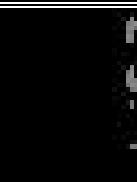 & $\overline{1}$ & 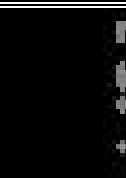 \\
\hline $\begin{array}{l}\text { Hole } \\
\text { (h1) }\end{array}$ & & & & \\
\hline & DSR & $95.70 \%$ & $95.62 \%$ & $95.43 \%$ \\
\hline & TPR & $48.95 \%$ & $56.58 \%$ & $55.18 \%$ \\
\hline
\end{tabular}

Fig. 7. Effect of the number of randomly located partitions as the locating process randomly picks up a certain number of pairs of $(\mathrm{x}, \mathrm{y})$ coordinates to locate a partition (see Section 3.2(B), step 4). 
and less than 5\%, respectively.

Fig. 7 shows the results when the choices of $r$ are 9, 16 and 25 for dot-patterned fabric.

In Fig. 6(a), the average DSR is shown to very stable, around 95\%, across the variation in the number of randomly located partitions. The TPR increases from $49.30 \%$ to $69.50 \%$ when $r$ rises from 5 to 60 . The FPR remains relatively stable at $4 \%$ at around 25 along the range of 5 and 60. The increase in $r$ does cause an increasing noise effect in the detection. For example, when $r \geq 20$, noise appears in the final result of some defect types such as Thick Bar in Fig. 7. In the third row of Fig. 7, noise appears only in Thick Bar (tt1) because its defective area is too large (around 1/4 of the image). Therefore, when $r$ is high, a defect-free partition will have a relatively high probability of matching a defective partition. Thus, defect-free partitions can gain a number of Elo points by matching with those defective partitions. As a result, many defect-free partitions are misclassified as light defects. It can be seen that $r=16$ is a good trade-off for the dot-patterned fabric, for which the $r$ is very close to 15 .

\section{c) Effect of the $w$-variable}

The $w$-variable determines the number of Elo points to be gained or lost per match.

If the difference in original Elo points between partitions $A$ and $B$ (i.e., $E_{a}-E_{b}$ before the match) is larger than $w$-variable, then the ER method will predict this match as a "must win" for partition $A$. Hence, partition $A$ will gain a low number of Elo points if it truly wins this match; otherwise, it will lose a large number of Elo points for the loss of this match. In 
other words, the $w$ - variable is capable of strengthening the small differences between the partitions in the image. The advantage of using a small $w$-variable is to intensify the small differences between any two partitions. Its shortcoming is that it may overemphasise the differences, sometimes leading the ER method to mistakenly treat a pattern as a defect. Thus, in fabric inspection, a small $w$ - variable should be used if the contrast in a pattern is large. Conversely, a large $w$-variable should be used for a low-contrast pattern.

We set the original $w$-variable to 400 , but it can be arbitrarily chosen. To study how this affects the inspection results, Fig. 8 illustrates the effect of $w$-variable in (2) on dot-, star- and box-patterned fabrics. In Fig. 8(a) of the dot-patterned fabric, the DSR increases from $92.75 \%$ at $w=100$ and remains around $95 \%$ after $w \geq 200$. TPR and FPR behave differently from the DSR in that both start at higher rates at $w=100(\mathrm{TPR}=69.43 \%, \mathrm{FPR}=6.85 \%)$ and then decrease to lower rates and stabilize when $w \geq 200$ (TPR between $62.02 \%$ and $64.80 \%$, FPR between 3.01\% and 3.65). The box-patterned fabric in Fig. 8(c) has similar but more stable performance of DSR, TPR and FPR (all rates appear stable for all choices of $w$-variable)

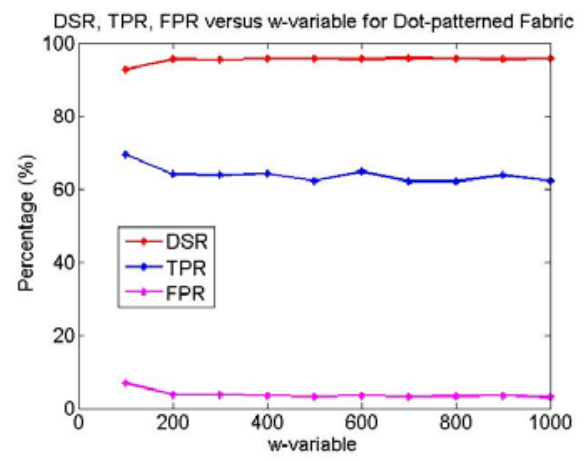

(a) dot-patterned

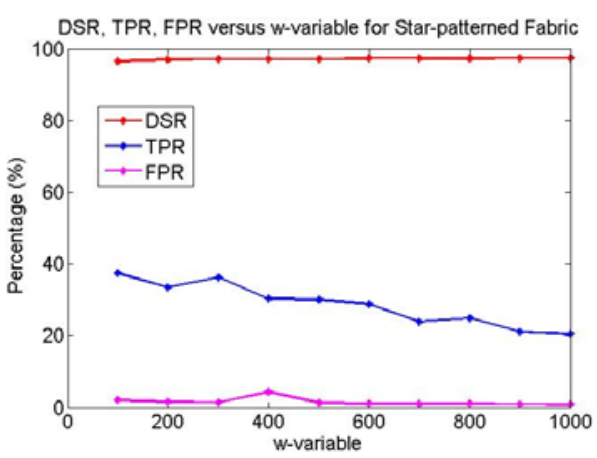

(b) star-patterned

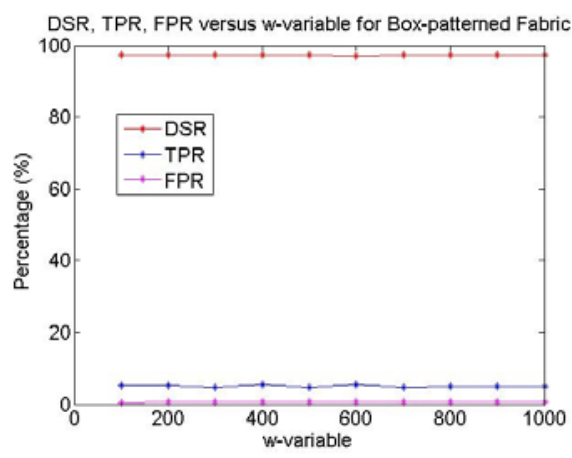

(c) box-patterned

Fig. 8. DSR, TPR and FPR versus $w$ - variable for (a) dot-patterned, (b) star-patterned and (c) box-patterned fabrics. 
compared with the dot-patterned fabric. The stable performance is actually due to the low contrast of the dot- and box-patterned fabrics. If $w$-variable is set to equal 100 , it will overemphasise the differences between any two partitions and the ER method will mistakenly treat a dot or box pattern as a defect. This problem can be immediately resolved by using a large $w$-variable. Therefore, all three measurement matrices stabilise with a large $w-$ variable at 400 in the dot- and box-patterned fabrics.

In Fig. $8(b)$ of the star-patterned fabric, the contrast is very large (the star pattern is completely white and the background region is completely dark) so that the ER method easily misclassifies the star pattern as a defect. The best TPR is $37.49 \%$ at $w=100$, whereas the later values of TPR decrease at $w>100$. Therefore, a small $w$-variable (i.e., 100) can provide a more accurate result in terms of TPR.

\section{d) Effect of the constant $K$}

The value of $K$ is the maximum or minimum number of Elo points of a player at a different skill level that can be gained or lost in a single match. It also relates to the speed at which a partition gains or loses a certain number of Elo points. In Fig. 9, the effect of K behaves similarly to the effect of the $w$-variable. $K$ acts like a multiplying factor to the $w-$ variable in the ER method. A partition can gain or lose a large number of Elo points if the difference between two partitions is greater than $w$-variable. If a large $K$ is also used, a 


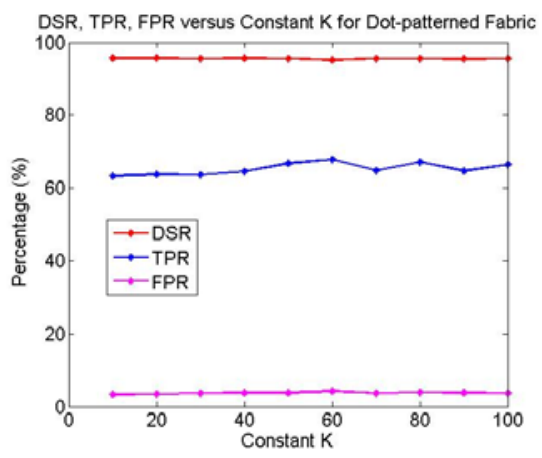

(a) dot-patterned

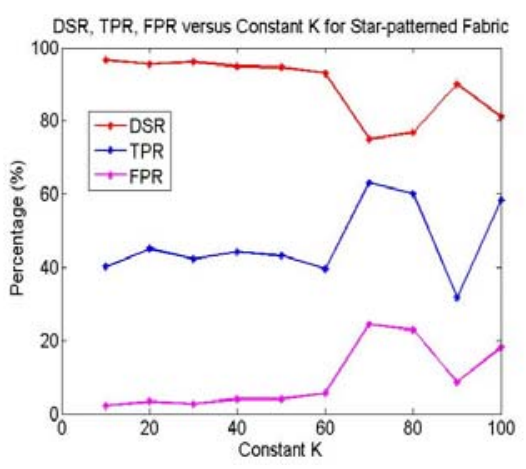

(b) star-patterned

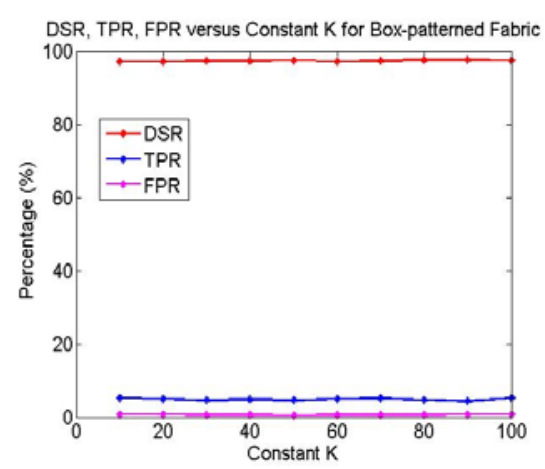

(c) box-patterned

Fig. 9. DSR, TPR and FPR versus constant $K$ for (a) dot-patterned, (b) star-patterned and (c) box-patterned fabrics.

partition will quickly gain or lose enough Elo points that the difference between this partition and other partitions will exceed $w$ - variable.

Fig. 9 shows the effect of $K$ on the dot-, star- and box-patterned fabrics. In Fig. 9(a), the DSR and FPR are very stable, at about $95 \%$ and $3.5 \%$ between 10 and 100 , respectively, whereas TPR fluctuates with a lower bound of $63.23 \%$ and an upper bound of $67.85 \%$ along the range of 10 to 100 . The values between 10 and 30 are very stable, indicating that $K=16$ is reasonable. Fig. 9(c) shows similar results for the box-patterned fabric. When a large $w-$ variable $(w=400)$ is used for either the dot- or box-patterned fabric, $\mathrm{K}$ has no effect on the DSR, TPR and FPR. Even if a K as large as 100 is used, it is still not high enough for a partition to gain or lose enough Elo points such that the differences between this partition and other partitions exceeds the designated $w$-variable. In addition, it is not reasonable to try an extremely large $K=100000$ because the contrast in the dot- and box-patterned fabrics is low. Therefore, a large $w$ - variable could prevent the overemphasis of the difference between partitions. Trying a large $K$ here means it will outweigh the effort of the setting the $w-$ 
variable. In summary, $K$ is found to be arbitrary as long as it does not exceed 100 . Therefore, 16 is chosen ast the initial $\mathrm{K}$, as suggested by Elo [23].

Contrastingly, when the $\mathrm{K}$ is large in the star-patterned fabric, the values of DSR, TPR and FPR become unstable (Fig. 9(b)). Therefore, a relatively small $w$ - variable is required for star-patterned fabrics $(w=100)$. If a relatively large $\mathrm{K}$ is used here, a partition can quickly gain or lose enough Elo points such that the difference between this partition and other partitions will exceed the $w$-variable. However, noise also accompanies a large $K$, which increases the FPR and decreases the DSR due to overemphasis of the difference between partitions. $K$ is set to 10 for the star-patterned fabric because this value performs relatively well and the DSR, TPR and FPR are all stable.

\subsection{Overall results of the ER method}

From Section 4.1 (a)-(d), the optimised sets of values for partition size $m \times n$, numbers randomly located in partitions $r, w-$ variable and constant $K,\{\{m \times n\}, r, w, K\}$, are $\{7 \times$ $4,15,400,16\},\{7 \times 4,40,100,10\}$ and $\{10 \times 6,15,400,16\}$ for the dot-, star-, boxpatterned fabrics, respectively.

\section{a) Numerical and graphical results}

Tables 3, 4 and 5 tabulate the numerical results of each defect type in dot-, star- and boxpatterned fabric images with two performance evaluation steps. The results of the recent fabric inspection method WGIS [18] are compared with those of the ER method. This is the first time, 
to our knowledge, that the WGIS method has been be tested on star- and box-patterned fabric images. The performance of other recent fabric inspection methods, such as the BB, RB and ID methods, on all three patterned fabrics can be obtained from our previous report [22].

It must be noted that the final total number of pixels in a resultant image in the ER method is 3538, which is lower than that of the WGIS method with its 65536 pixels. In the first step, the percentage white pixels of the total pixels obtained in the ER method for the dot-, star- and box-patterned fabric defect-free images are $0.11 \%\left(\frac{3.87}{3538}\right), 0$ and 0 white pixels, whereas those obtained with the WGIS method are $28.43 \%\left(\frac{18635}{65536}\right), 3.57 \%\left(\frac{2339}{65536}\right)$ and $25.13 \%\left(\frac{16470}{65536}\right)$ white pixels, respectively. This shows that the ER method performs well because it generates fewer white pixels for defect-free images.

The ER method also results in fewer white pixels than the WGIS method in the defective images of all three types of patterned fabrics. For the dot-patterned fabric samples in the ER method, the percentage of white pixels of the total pixels in the resultant images are between $\sim 0 \%\left(\frac{0.33}{3538}\right)$ and $23.58 \%\left(\frac{834.33}{3538}\right)$ from Table 3 (dot-patterned), between $1.23 \%\left(\frac{43.4}{3538}\right)$ and $4.53 \%\left(\frac{160.2}{3538}\right)$ from Table 4 (star-patterned) and within 0 (or $0 \%$ ) and $4.54 \%\left(\frac{147.4}{3245}\right)$ from Table 5 (box-patterned). In comparison, the WGIS method gives ranges between $11.19 \%\left(\frac{7332.66}{65536}\right)$ and $25.65 \%\left(\frac{16812}{65536}\right)$ from Table 3, 3.5\% $\left(\frac{2295}{65536}\right)$ and $3.65 \%\left(\frac{2388.9}{65536}\right)$ from Table 4 and $2.47 \%\left(\frac{1617}{65536}\right)$ and $24.84 \%\left(\frac{16277}{65536}\right)$ from Table 5. As a result, the ER method obtains a higher percentage of white pixels and demonstrates stronger discriminative power to detect defects in the first step. 
The detailed measurement metrics are obtained in the second step. Here, the ER method obtains overall results of $96.89 \%$ DSR, $42.40 \%$ TPR, 0.04\% FPR, 34.91\% PPV and 97.32\% NPV for the dot-patterned fabric (Table 3), whereas the WGIS method generates overall results of $77.04 \%$ DSR, $58.10 \%$ TPR, 0.17\% FPR, 12.82\% PPV and 97.98\% NPV. The higher value of PPV means that the ER method is more accurate when detecting defective regions, and the lower values of FPR and NPV mean that the method is more accurate when detecting defectfree regions. A higher DSR means better overall performance in detection. Fig. 10 depicts the detection results of the WGIS and ER methods compared with the ground-truth images. The ER method detects the light defect, Knots (first row) and Loose Pick (ninth row), more accurately than the WGIS method. For the dark defects, i.e., Thin Bar, Thick Bar, Netting Multiple, Broken End, Hole, Oil Warp, Oil Weft and Miss Pick, the ER method performs better than the WGIS method with more accurate locations and more finely detected defect shapes.

From Table 4 of the star-patterned fabric, the ER method shows better overall results than the WGIS method in the metrics of DSR (98.82\% versus $95.73 \%)$, TPR (32.93\% versus $1.2 \%)$ and PPV (19.70\% versus $1.22 \%)$ but poorer results for FPR (7.71\% versus $3.58 \%)$ and NPV (99.12\% versus 98.51\%). In Fig. 11, the WGIS method only provides white dots, which are not a satisfactory visualised result, compared with the ER method. Table 5 shows the results from the box-patterned fabric, in which the ER method shows higher rates than the WGIS method in the overall results of DSR (95.51\% versus $49.91 \%)$ and PPV (15.84\% versus $2.09 \%)$ and lower rates for TPR (7.80\% versus $35.31 \%)$, FPR (1.39\% versus $24.88 \%)$ and NPV 
(96.80\% versus $98.89 \%$ ). Fig. 12 illustrates that the WGIS method offers many extra white

boxes, which are falsely detected as defective regions. This is also why the WGIS method

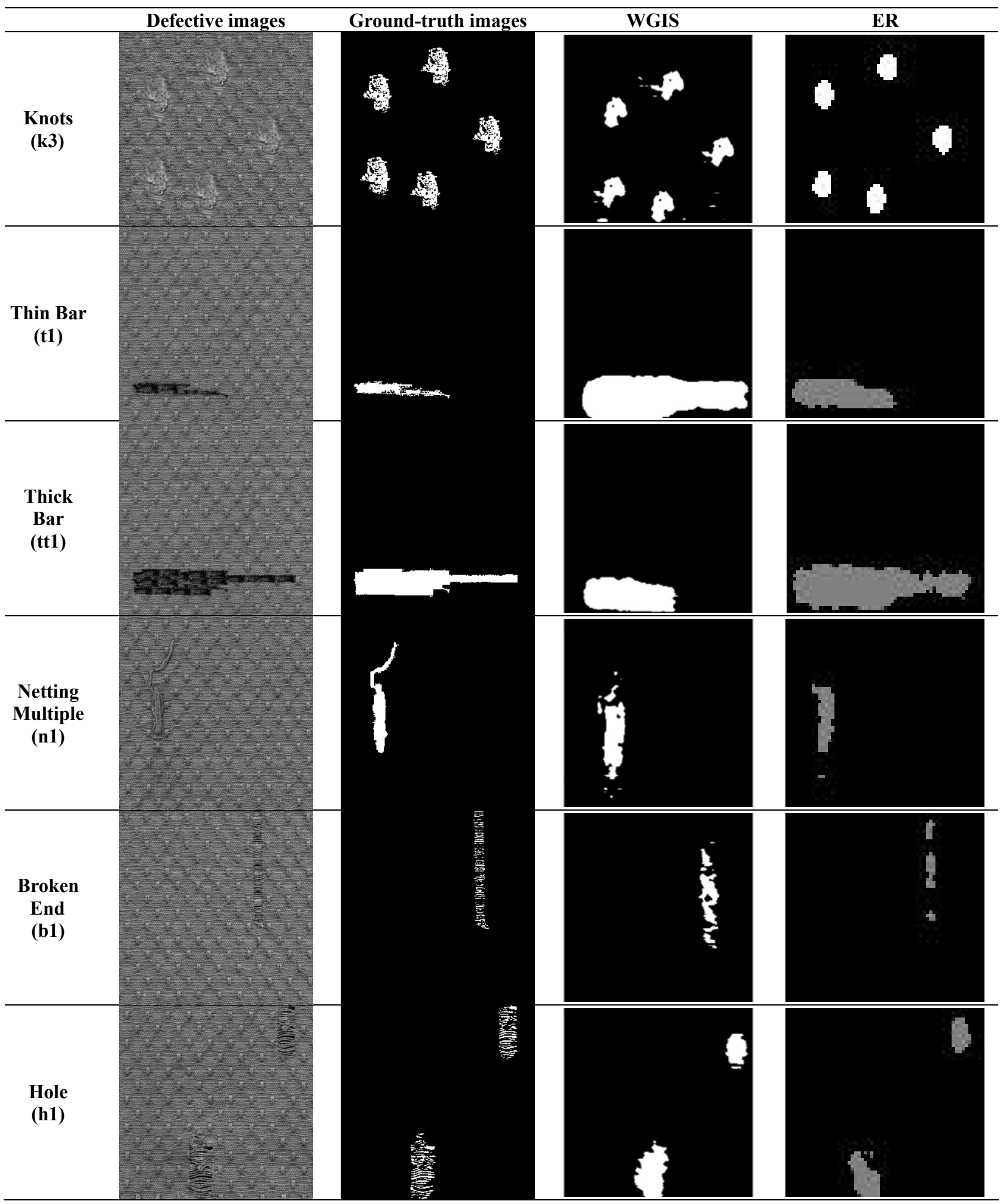




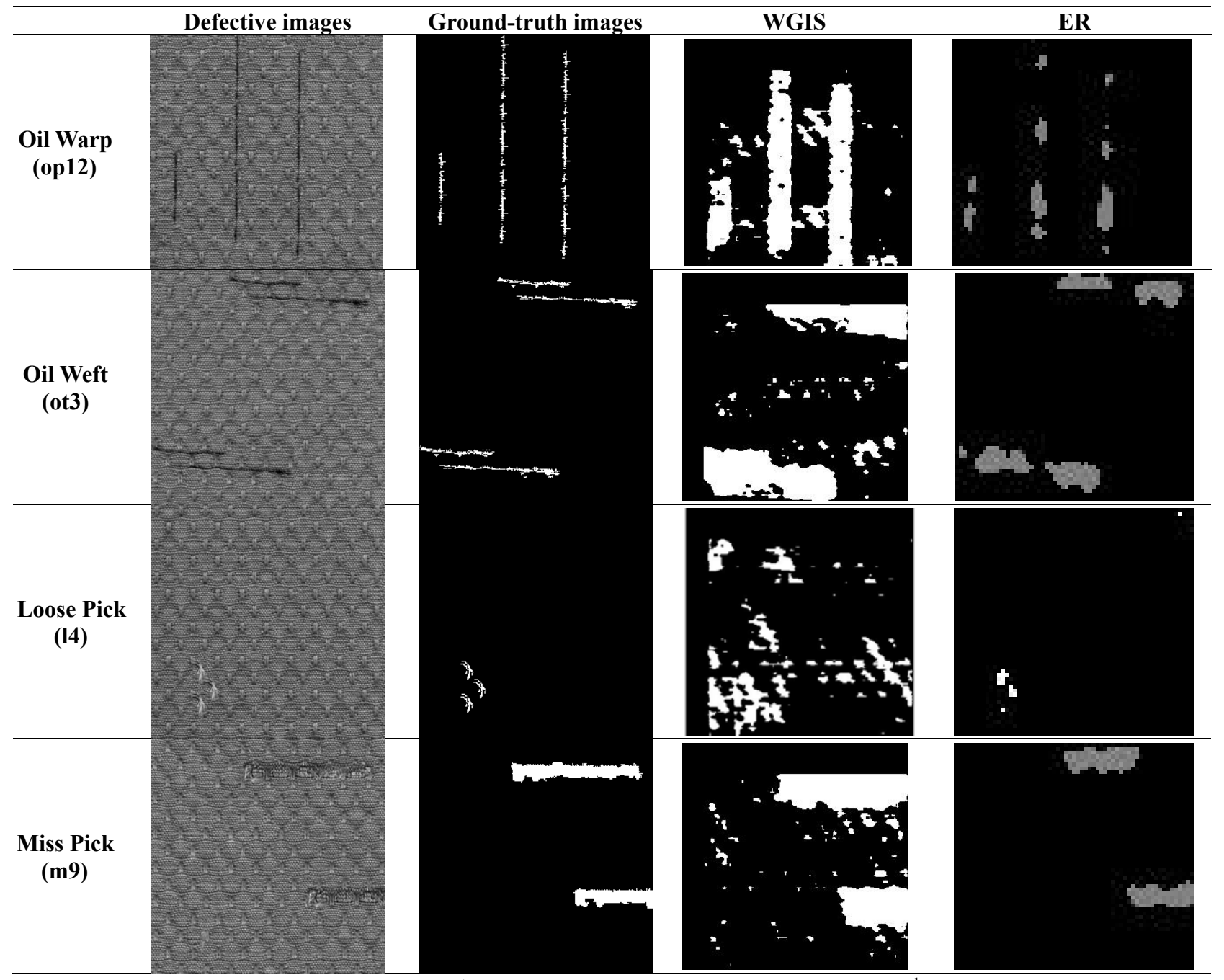

Fig. 10. Dot-patterned fabric: ( $1^{\text {st }}$ column) Defective sample names; ( $2^{\text {nd }}$ column $)$ Defective images; ( $3^{\text {rd }}$ column) Ground-truth image; $\left(4^{\text {th }}\right.$ column $)$ Detection results of WGIS method; $\left(5^{\text {th }}\right.$ column) Detection results of ER method. Partition size $=7 \times 4$, Number of randomly located partitions $=16, w-$ variable $=400$, Constant $K=16$. Remark: Threshold light $_{\text {value }}$ in the ER method is reduced by $5 \%$ for Loose Pick.

obtains a high TPR (35.31\%) and FPR (24.88\%). The ER method is found to be incapable of detecting the defects of Netting Multiple and Hole, as indicated by Fig. 12. 


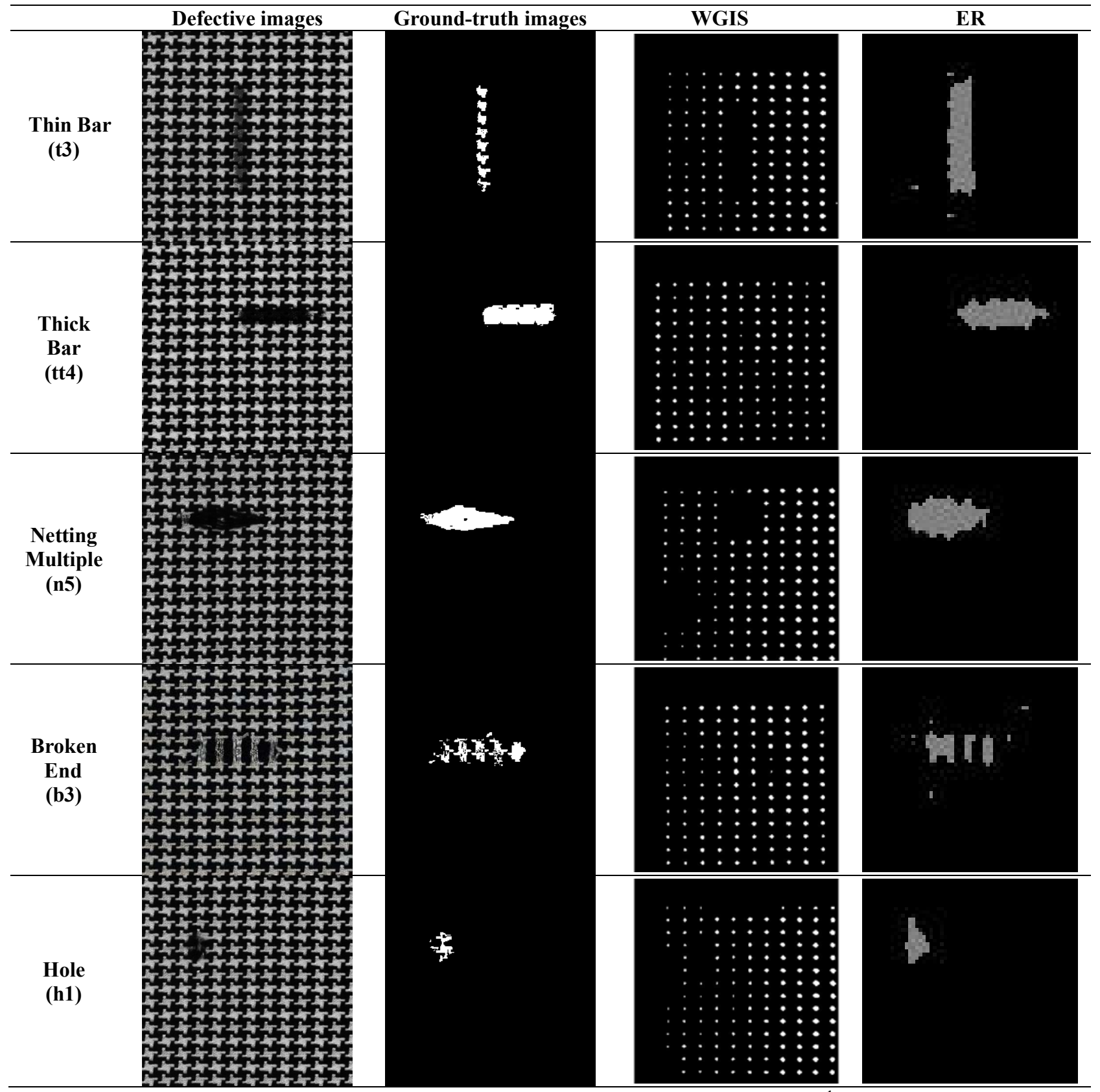

Fig. 11. Star-patterned fabric: ( $1^{\text {st }}$ column) Defective sample names; $\left(2^{\text {nd }}\right.$ column $)$ Defective images; ( $3^{\text {rd }}$ column) Ground-truth image; ( $4^{\text {th }}$ column$)$ Detection results of WGIS method; ( $5^{\text {th }}$ column) Detection results of ER method. Partition size $=7 \times 4$, Number of randomly located partitions $=40, w-$ variable $=100$, Constant $K=10$. 
Table 3

Numerical results of each defect type: WGIS and ER methods on dot-patterned fabric images

\begin{tabular}{|c|c|c|c|c|c|c|c|}
\hline & Avg defective pixels (No.) & $\operatorname{DSR}(\%)$ & TPR (\%) & $\operatorname{FPR}(\%)$ & PPV (\%) & NPV (\%) & Method \\
\hline $\begin{array}{l}\text { Defect-free } \\
\text { (110) }\end{array}$ & 18635 & 71.57 & $\mathrm{~N} / \mathrm{A}$ & $\mathrm{N} / \mathrm{A}$ & $\mathrm{N} / \mathrm{A}$ & $\mathrm{N} / \mathrm{A}$ & WGIS \\
\hline \multirow{2}{*}{ Knots (12) } & 16812.00 & 73.55 & 38.61 & 0.25 & 4.89 & 97.10 & WGIS \\
\hline & 115.08 & 97.07 & 54.35 & 0.01 & 66.88 & 98.10 & $\mathbf{E R}$ \\
\hline \multirow{2}{*}{ Thin Bar (12) } & 11360.21 & 83.39 & 66.69 & 0.16 & 10.66 & 98.64 & WGIS \\
\hline & 333.08 & 92.50 & 81.22 & 0.07 & 26.81 & 99.30 & $\mathbf{E R}$ \\
\hline \multirow{2}{*}{ Thick Bar (12) } & 15284.02 & 81.49 & 71.66 & 0.17 & 33.18 & 95.58 & WGIS \\
\hline & 834.33 & 84.76 & 84.94 & 0.15 & 49.46 & 96.19 & ER \\
\hline \multirow{2}{*}{$\begin{array}{l}\text { Netting } \\
\text { Multiple (12) }\end{array}$} & 13055.00 & 81.06 & 62.92 & 0.18 & 14.10 & 97.73 & WGIS \\
\hline & 92.00 & 96.41 & 43.79 & 0.01 & 80.95 & 97.79 & ER \\
\hline \multirow{2}{*}{$\begin{array}{l}\text { Broken End } \\
\text { (12) }\end{array}$} & 14890.23 & 80.04 & 54.93 & 0.18 & 25.51 & 93.90 & WGIS \\
\hline & 236.75 & 91.69 & 32.27 & 0.01 & 56.25 & 91.90 & ER \\
\hline \multirow{2}{*}{ Hole (12) } & 11975.31 & 83.06 & 75.13 & 0.17 & 10.92 & 99.15 & WGIS \\
\hline & 232.00 & 94.49 & 69.21 & 0.05 & 30.63 & 98.94 & ER \\
\hline \multirow{2}{*}{ Oil Warp (12) } & 10450.53 & 83.88 & 42.85 & 0.16 & 2.43 & 99.34 & WGIS \\
\hline & 87.75 & 97.03 & 24.58 & 0.02 & 8.84 & 99.20 & ER \\
\hline \multirow{2}{*}{ Loose Pick (12) } & 7332.66 & 88.72 & 36.70 & 0.11 & 1.08 & 99.76 & WGIS \\
\hline & 0.33 & 99.62 & 0.00 & 0.00 & 0.00 & 99.63 & ER \\
\hline \multirow{2}{*}{ Miss Pick (12) } & 14524.36 & 81.48 & 74.76 & 0.18 & 21.59 & 98.14 & WGIS \\
\hline & 137.00 & 90.10 & 8.74 & 0.03 & 17.81 & 93.02 & ER \\
\hline \multirow{2}{*}{ Overall } & $\mathrm{N} / \mathrm{A}$ & 77.04 & 58.10 & 0.17 & 12.82 & 97.98 & WGIS \\
\hline & N/A & 96.89 & 42.40 & 0.04 & 34.91 & 97.32 & ER \\
\hline
\end{tabular}

Remark: The total number of pixels of resultant images of the WGIS and ER methods are $256 \times 256=65536$ and $58 \times 61=3538$, respectively. The numbers in brackets indicate the number of samples. 
Table 4

Numerical results of each defect type: WGIS and ER methods on star-patterned fabric images

\begin{tabular}{lccccccc}
\hline \hline & Avg defective pixels (No.) & DSR (\%) & TPR (\%) & FPR (\%) & PPV (\%) & NPV (\%) & Method \\
\hline \hline \multirow{2}{*}{ Defect-free (30) } & 2339.6 & 96.43 & N/A & N/A & N/A & N/A & WGIS \\
\cline { 2 - 8 } & 0 & 100 & N/A & N/A & N/A & N/A & ER \\
\hline \multirow{2}{*}{ Thin Bar (5) } & 2370 & 95.42 & 0 & 3.65 & 0 & 99.00 & WGIS \\
\cline { 2 - 8 } & 120.6 & 96.72 & 45.47 & 2.83 & 12.50 & 99.45 & ER \\
\hline \multirow{2}{*}{ Thick Bar (6) } & 2295 & 93.40 & 4.37 & 3.44 & 5.09 & 96.63 & WGIS \\
\cline { 2 - 8 } Netting & 160.2 & 97.30 & 69.52 & 1.67 & 54.52 & 98.81 & ER \\
\cline { 2 - 8 } Multiple (5) & 2345 & 94.76 & 1.03 & 3.61 & 0.88 & 98.25 & WGIS \\
\multirow{2}{*}{ Broken End (5) } & 46.8 & 97.76 & 16.42 & 0.82 & 12.61 & 98.54 & ER \\
\hline \multirow{2}{*}{ Hole (5) } & 2318 & 95.74 & 0.26 & 3.56 & 0.09 & 99.24 & WGIS \\
\cline { 2 - 8 } & 43.4 & 98.13 & 8.79 & 1.16 & 7.17 & 99.27 & ER \\
\hline \multirow{2}{*}{ Overall } & 2388.9 & 95.82 & 0.34 & 3.66 & 0.05 & 99.45 & WGIS \\
\cline { 2 - 8 } & 49 & 95.32 & 24.47 & 1.23 & 11.68 & 99.54 & ER \\
\hline \hline
\end{tabular}

Remark: The total number of pixels of resultant images of the WGIS and ER methods are $256 \times 256=65536$ and $58 \times 61=3538$, respectively. The numbers in brackets indicate the number of samples.

Table 5

Numerical results of each defect type: WGIS and ER methods on box-patterned fabric images

\begin{tabular}{lccccccc}
\hline \hline & Avg defective pixels (No.) & DSR (\%) & TPR (\%) & FPR (\%) & PPV (\%) & NPV (\%) & Method \\
\hline \hline \multirow{2}{*}{ Defect-free (25) } & 16470 & 25.13 & N/A & N/A & N/A & N/A & WGIS \\
\cline { 2 - 7 } & 0 & 100 & N/A & N/A & N/A & N/A & ER \\
\hline \multirow{2}{*}{ Thin Bar (5) } & 15870 & 75.33 & 26.90 & 24.20 & 1.02 & 99.07 & WGIS \\
\cline { 2 - 8 } & 147.4 & 93.41 & 5.84 & 4.51 & 2.36 & 97.68 & ER \\
\hline \multirow{2}{*}{ Thick Bar (5) } & 16277 & 75.32 & 49.08 & 24.24 & 4.30 & 98.77 & WGIS \\
\cline { 2 - 8 } Netting & 86.4 & 95.43 & 22.76 & 1.68 & 42.40 & 96.93 & ER \\
\cline { 2 - 8 } Multiple (5) & 1684 & 73.77 & 33.00 & 25.68 & 1.28 & 98.87 & WGIS \\
\hline \multirow{2}{*}{ Broken End (5) } & 1.4 & 95.77 & 0.15 & 0.04 & 4 & 95.81 & ER \\
\hline \multirow{2}{*}{ Hole (5) } & 1617 & 74.85 & 36.39 & 24.43 & 2.94 & 98.40 & WGIS \\
\cline { 2 - 8 } & 34.6 & 95.27 & 10.26 & 0.69 & 30.43 & 95.88 & ER \\
\hline \hline \multirow{2}{*}{ Overall } & 1675 & 74.17 & 31.17 & 25.52 & 0.92 & 99.31 & WGIS \\
\cline { 2 - 8 } & 0.8 & 97.66 & 0 & 0.03 & 0 & 97.69 & ER \\
\hline \hline
\end{tabular}

Remark: The total number of pixels of resultant images of the WGIS and ER methods are $256 \times 256=65536$ and $55 \times 59=3538$, respectively. The numbers in brackets indicate the number of samples. 


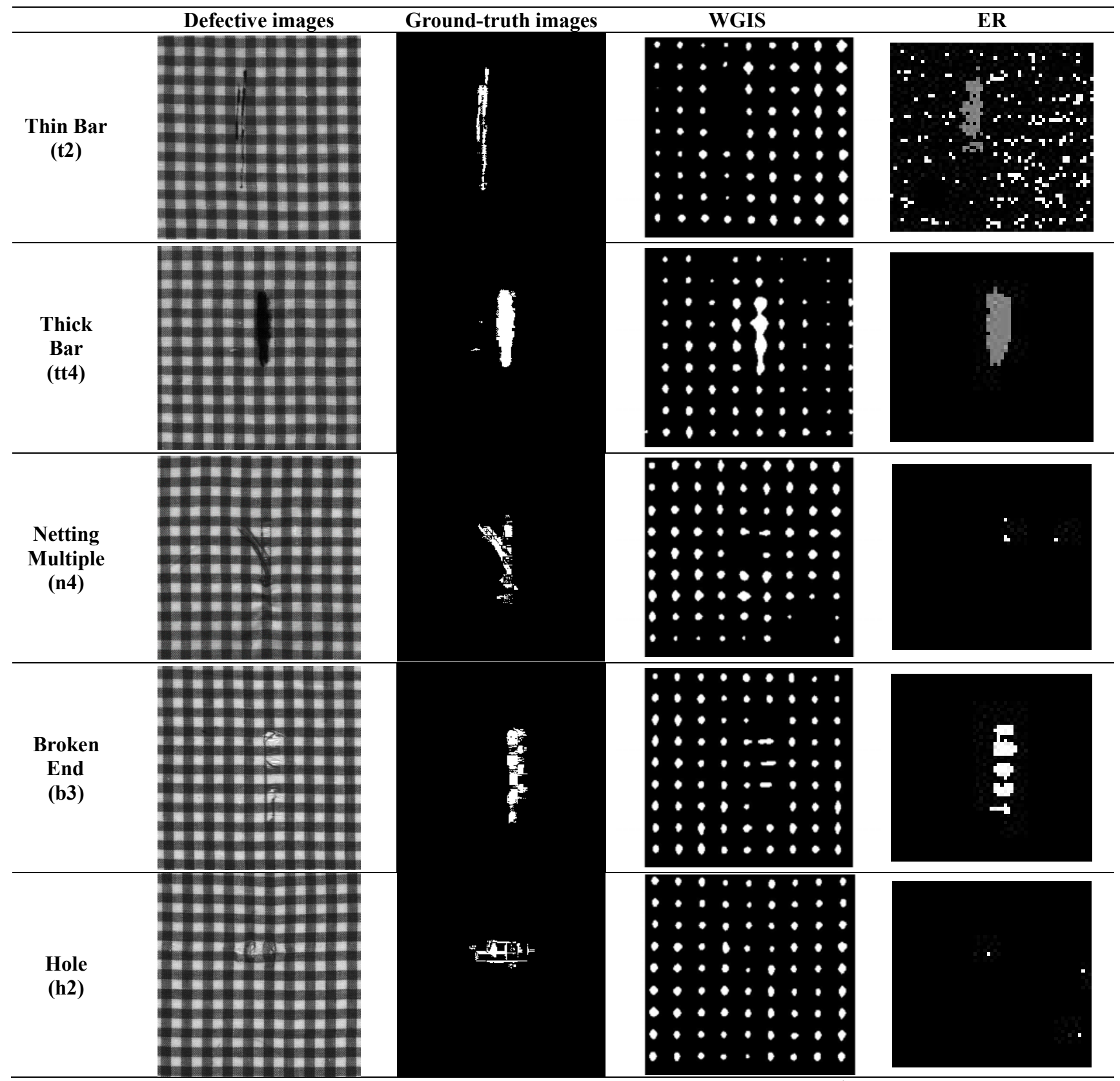

Fig. 12. Box-patterned fabric: $\left(1^{\text {st }}\right.$ column $)$ Defective sample names; $\left(2^{\text {nd }}\right.$ column $)$ Defective images; ( $3^{\text {rd }}$ column) Ground-truth image; $\left(4^{\text {th }}\right.$ column$)$ Detection results of WGIS method; $\left(5^{\text {th }}\right.$ column) Detection results of ER method. Partition size $=10 \times 6$, Number of randomly located partitions $=25 . w-$ variable $=400$, Constant $K=16$. The median filtering in the last step is skipped for this box-patterned fabric. 


\section{b) TPR-FPR graphs with optimised parameters}

A further detailed comparison with TPR-FPR graphs [22] between the BB, RB, ID, WGIS and ER methods is shown in Fig. 13. All methods were evaluated on the dot-, star- and box-patterned fabric databases in [21]. Similar to that in an ROC graph, a point located closer to the top left corner of the TPR-FPR graphs is regarded as an optimised result. The TPR-FPR graphs are formulated by the TPR and FPR values of each defective sample of the dot-, starand box-patterned fabrics by the BB, RB, ID, WGIS and ER methods. This TPR-FPR graph can help to evaluate how each method performs on each particular defect type. Only dotpatterned fabric has the Knots defect. The blue dots in Fig. 13(a) clearly show that the TPRFPR points of the ER method are close to the top left corner of the graph than those of the BB, RB and WGIS methods. For the dot-patterned fabric, the ER method obviously outperforms the BB, RB and WGIS methods for each defect type. In regard to the star-patterned fabric, the ER method (blue diamonds) demonstrates superiority in the Thin Bar (Fig. 13(b)), Thick Bar (Fig. 13(c)), Netting Multiple (Fig. 13(d)), Broken End (Fig. 13(e)) and Hole (Fig. 13(f)) defects. Most of the TPR-FPR points of the BB, RB and WGIS methods, shown as cyan, magenta and red diamonds, are located at the bottom left corner of the plots, indicating both low TPR and low FPR. This is also due to the complete darkness in the final resultant images once all noise is removed. For the box-patterned fabric, most methods do not performs as well as in the previous two patterned fabrics. The ER method performs slightly better than the WGIS method and much better than the BB and RB methods in the Thick bar defect (ER: blue boxes 
with lower FPR and higher TPR in Fig. 13(c)) and Broken End (Fig. 13(e)). However, the ER method performs worse than the WGIS method in the Thin Bar (Fig. 13(b)), Netting Multiple

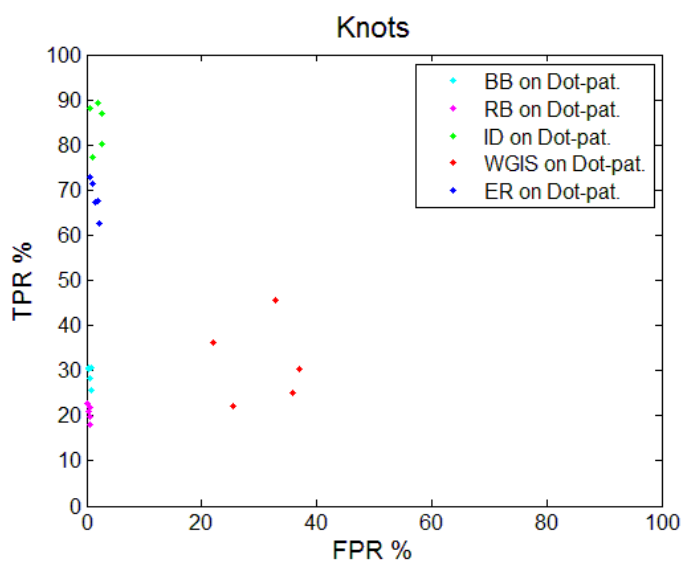

(a)

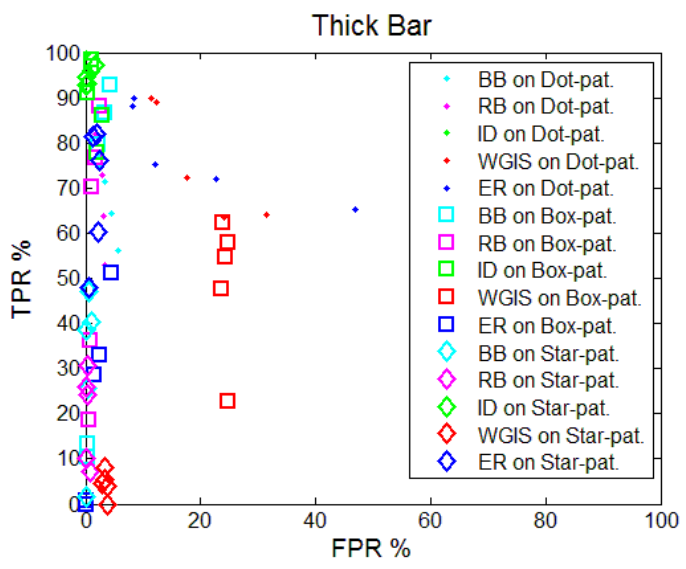

(c)

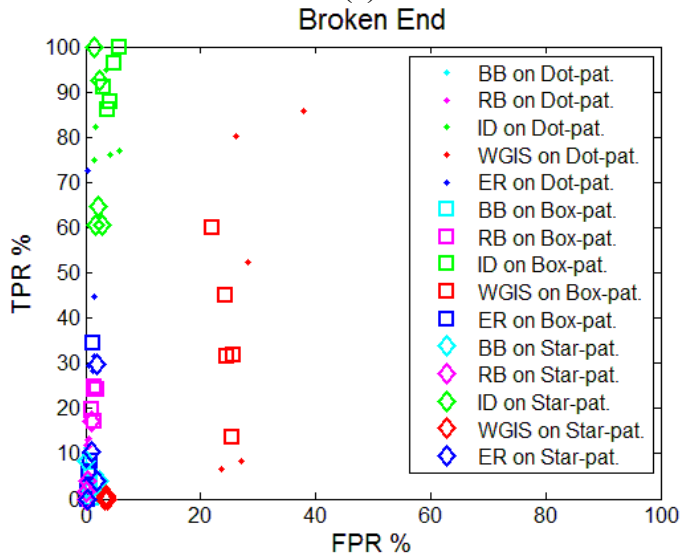

(e)

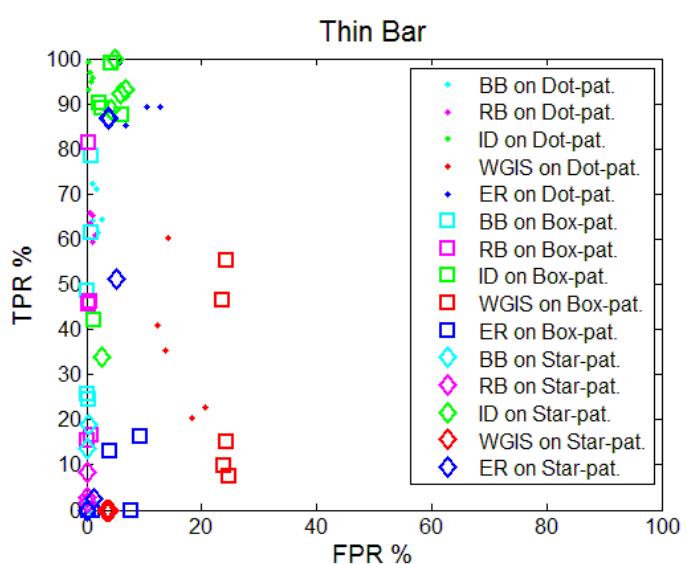

(b)

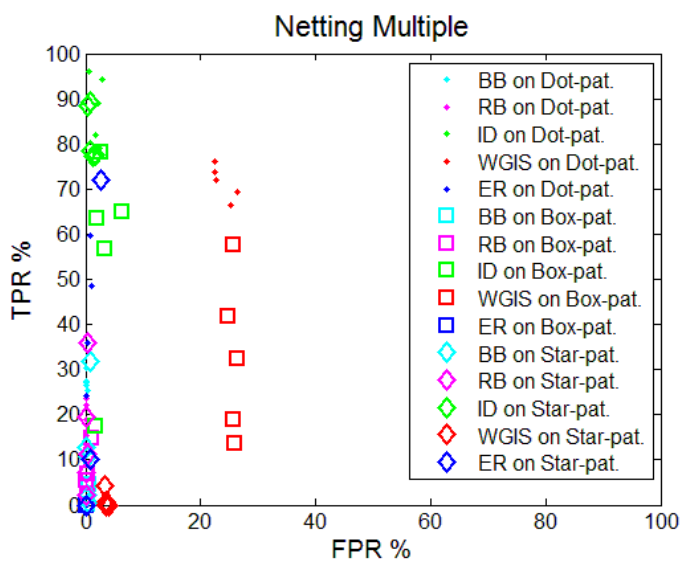

(d)

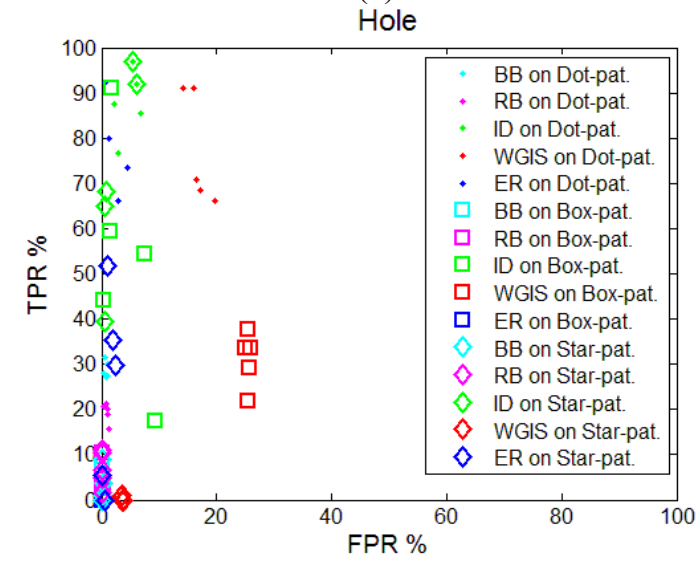

(f)

Fig. 13. FPR-TPR graphs of six defect types of dot-, box- and star-patterned fabric samples: (a) Knots, (b) Thin Bar, (c) Thick Bar, (d) Netting Multiple, (e) Broken End and (f) Hole. BB method (cyan); RB method (magenta); ID method (green); WGIS method (red); ER method (blue). Dot-pattern (circles); box-pattern (squares); star-pattern (diamonds). 
(Fig. 13(d)), Broken End (Fig. 13(e)) and Hole defects (Fig. 13(f)), with most of the blue boxes found at the bottom left in those plots. Compared with the corresponding red boxes of the WGIS method, they have higher a TPR and a higher FPR, indicating that the ER method still has room for improvement in the inspection of box-patterned fabric. In short, although the ID method generated better TPR-FPR points than the ER method, it is a semi-supervised approach that requires a defective sample and a defect-free sample for training. On the contrary, the WGIS, BB, RB and ER methods as a supervised approach employed only defect-free samples for training that reveals more close to the real inspection situation because defects are not predictable.

\section{CONCLUSION}

This paper presents a new method of patterned fabric inspection called the ER method, in which the detection of defects is similar to carrying out fair matches in the spirit of good sportsmanship. The ER method achieved an overall $97.07 \%$ detection success rate based on 336 images from dot-, star- and box-patterned fabrics, compared with the evaluation of groundtruth images. The ER method depends on four parameters, partition size, the number of randomly located partitions, $w$ - variable and constant K. A study of their significance was carried out. The ER method performed well in the dot- and star-patterned fabrics, but it still has room for improvement in the box-patterned fabric. In the future, additional theoretical development involving game theory for matches as it relates to defect detection should be carried out. Such research will be beneficial for defect detection in the textile, tile, ceramics, 
wallpaper, aircraft window and printed circuit board industries and for the latest threedimensional printing technologies.

\section{Acknowledgements}

The second author was supported by a Hong Kong Baptist University Faculty Research

Grant/12-13/075. 

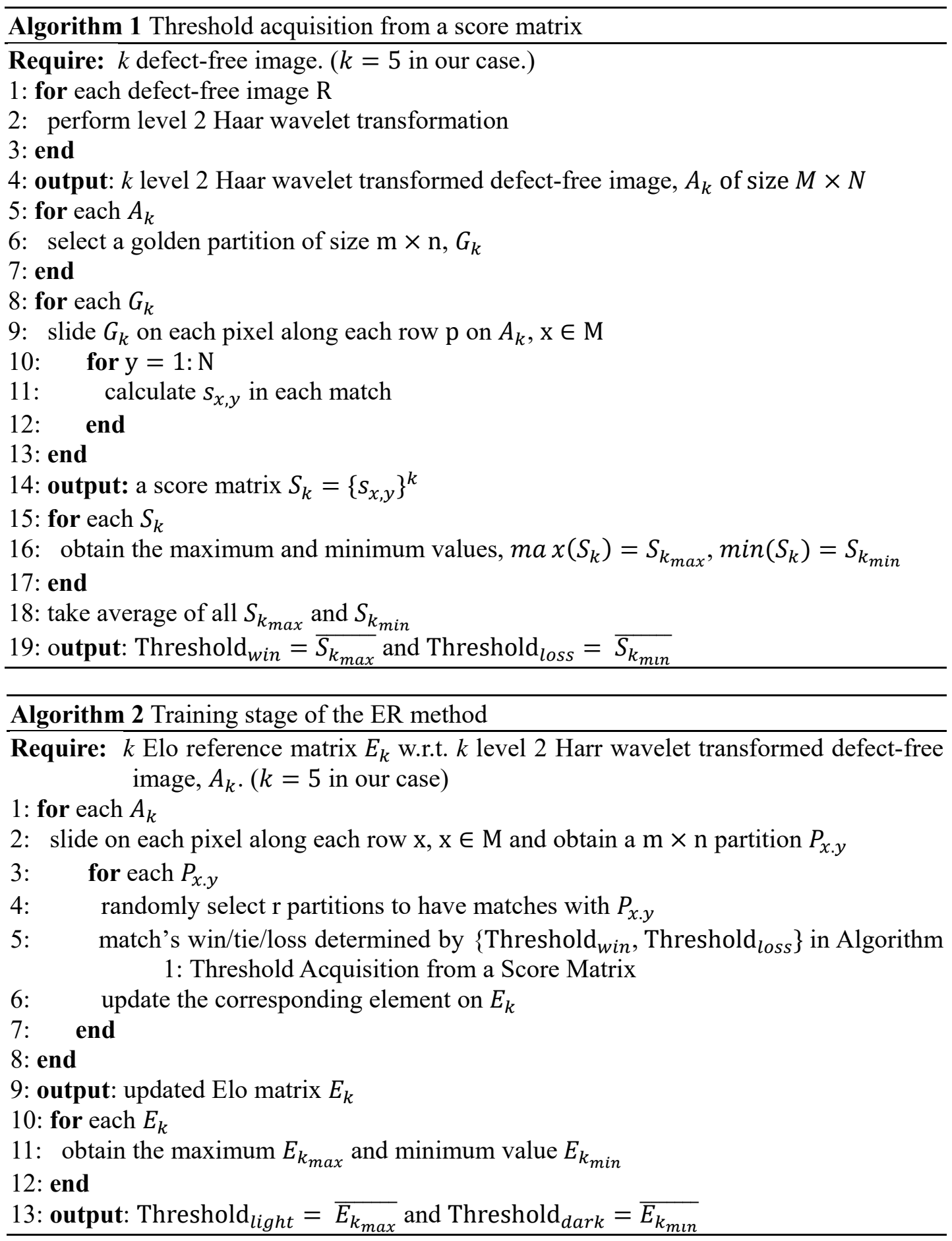


\section{REFERENCES}

[1] H.Y.T. Ngan, G.K.H. Pang and N.H.C. Yung, "Automated Fabric Defect Detection - A Review," Image and Vision Computing, vol. 29, no. 7, pp. 442-458, 2011.

[2] H.Y.T. Ngan, G.K.H. Pang and N.H.C. Yung, "Motif-based Defect Detection for Patterned Fabric," Pattern Recognition, vol. 41, no. 6, pp. 1878-1894, 2008.

[3] D-M. Tsai, M-C. Chen, W-C. Li and W-Y. Chiu, "A Fast Regularity Measure for Surface Defect Detection," Machine and Vision Application, vol. 23, pp. 869-886, 2012.

[4] H-G. Bu, J. Wang and X-B. Huang, "Fabric Defect Detection based on Multiple Fractal Features and Support Vector Data Description," Engineering Applications of Artificial Intelligence, vol. 22, pp. 224-235, 2009.

[5] K.L. Mak, P. Peng, K.F.C. Yiu, "Fabric Defect Detection using Morphological Filters," Image and Vision Computing, vol. 27, pp. 1585-1592, 2009.

[6] M.A. Aziz, A.S. Hagga and M.S. Sayed, "Fabric Defect Detection Algorithm using Morphological Processing and DCT," Proc. IEEE ICCSPA, pp. 1-4, 2013.

[7] C. H. Chan and G.K.H. Pang., "Fabric Defect Detection by Fourier Analysis," IEEE Trans. Industry Applications, vol.36, no.5, Sept/Oct 2000.

[8] D. Aiger and H. Talbot, "The Phase Only Transform for Unsupervised Surface Defect Detection," Proc. IEEE CVPR, pp. 295-302, 2010.

[9] D. Schneider, T. Holtermann, F. Neumann, A. Hehl, T. Aach and T. Gries, "A Vision Based System for High Precision Online Fabric Defect Detection," Proc. IEEE ICIEA, pp. 14941499, 2012.

[10] A. Kumar and G.K.H. Pang, "Defect Detection in Textured Materials Using Gabor Filters," IEEE Trans. Industry Applications, vol. 38, no.2, pp. 425-440, 2002.

[11] X.Z. Yang, G.K.H. Pang and N.H.C. Yung, "Discriminative fabric defect detection using directional wavelets," Optical Engineering, vol. 41, no. 12, pp. 3116-3126, 2002.

[12] X.Z. Yang, G.K.H. Pang and N.H.C. Yung, "Discriminative training approaches to fabric defect classification based on wavelet transform," Pattern Recognition, vol. 37, issue 5, pp. 889-899, 2004.

[13] H. Zuo, Y. Wang, X. Yang and X. Wang, "Fabric Defect Detection Based on Texture Enhancement," Proc. IEEE CISP, pp. 876-880, 2012.

[14] F.S. Cohen, Z.G. Fan, S. Attali, "Automated Inspection of Textile Fabric using Textural Models," IEEE Trans. Pattern Analysis \& Machine Intelligence, vol. 13, no. 8, pp. 803808, Aug 1991.

[15] J. Zhou, D. Semenovich, A. Sowmya and J. Wang, "Sparse Dictionary Reconstruction for Textile Defect Detection," Proc. IEEE ICMLA, pp. 21-26, 2012.

[16] Z. Kang, C. Yuan and Q. Yang, "The Fabric Defect Detection Technology Based on Wavelet Transform and Neural Network Convergence," Proc. IEEE ICIA, pp. 597-601, 2013.

[17] A. Bodnarova, M. Bennamoun, and K.K. Kubik, "Defect Defection in Textile Materials Based on Aspects on the HVS," Proc. IEEE Int'l Conf. SMC, pp. 4423-4428, 1998.

[18] H.Y.T. Ngan, G.K.H. Pang, S.P. Yung and M.K. Ng, "Wavelet based methods on Patterned Fabric Defect Detection," Pattern Recognition, vol. 38, issue 4, pp. 559-576, 2005.

[19] C.J. Kuo, and T. Su, "Gray relational analysis for recognizing fabric defects," Textile Research Journal, vol. 73, no. 5, pp. 461-465, 2003.

[20] H.Y.T. Ngan and G.K.H. Pang, "Novel Method for Patterned Fabric Inspection using Bollinger Bands," Optical Engineering, vol. 45, no. 8, Aug, 2006.

[21] H.Y.T. Ngan and G.K.H. Pang, "Regularity Analysis for Patterned Texture Inspection," IEEE Trans. Automation Science \& Engineering, vol. 6, no. 1, pp. 131-144, 2009. 
[22] M.K. Ng, H.Y.T. Ngan, X. Yuan and W. Zhang, "Patterned Fabric Inspection and Visualization by the Method of Image Decomposition," IEEE Trans. Automation Science \& Engineering, vol. 11, no. 3, pp. 943-947, 2014.

[23] A. E. Elo, The Rating of Chess Players, Past and Present, New York: Arco, 1978.

[24] P.C.H. Albers and H. de Varies, "Elo-rating as a Tool in the Sequential Estimation of Dominance Strengths", Animal Behaviour, vol. 61, pp. 489-490, 2001.

[25] H.Y.T. Ngan, G.K.H. Pang and N.H.C. Yung, "Performance Evaluation for Motif-based Patterned Texture Defect Detection," IEEE Trans. Automation Science \& Engineering, vol. 7, no. 1, pp. 58-72, 2010.

[26] Y. Li and X. Di, "Fabric Defect Detection using Wavelet Decomposition," Proc. IEEE CECNet, pp. 308-311, 2013.

[27] G. Stubl, J-L. Bouchot, P. Haslinger and B. Moser, "Discrepancy Norm as Fitness Function for Defect Detection on Regularly Textured Surfaces," LNCS, vol. 7476, pp. 428-437, 2012.

[28] J.L. Raheja, S. Kumar and A. Chaudhary, "Fabric Defect Detection based on GLCM and Gabor filter: A Comparison," Optik - Int'l J. Light and Electron Optics, vol. 124, no. 23, pp. 6469-6474, 2013.

[29] M. Li, Z. Deng and L. Wang, "Defect Detection of Patterned Fabric by Spectral Estimation Technique and Rough Set Classifier," Proc. IEEE GCIS, pp. 190-194, 2013. 


\section{VITAE}

COLIN S.C. TSANG is an undergraduate year-4 student in mathematics at Hong Kong Baptist University, China. He is supposed to obtain B.Sc. (Hons.) in mathematical science in 2015. He likes sport matches in daily life. His current research interests include surface defect detection, pattern recognition and image processing.

HENRY Y.T. NGAN obtained a B.Sc. degree in mathematics in 2001, a M. Phil. Degree in 2005 and a Ph.D. degree in 2008 in electrical and electronic engineering at The University of Hong Kong (HKU), China. He is currently a research assistant professor at the Department of Mathematics, Hong Kong Baptist University. Previously, he worked in the Laboratory for Intelligent Transportation Systems Research, HKU in 2010-2012 and the IARL, HKU in 2002-2008. He held visiting positions in Electric \& Electrical Engineering, the University of Sheffield, U.K. in 2012-2014, the CCIL, NEC, Japan and the Carnegie Mellon CyLab, Carnegie Mellon University, U.S. at Kobe, Japan in 2008-2009, and at the IAL, the University of British Columbia, Canada in 2006. He carried out industrial and consultancy projects for Hong Kong ASTRI and CFM Management Company Ltd., H.K. in 2010-2011. His current research interests include pattern recognition application on anomalies detection, large-scale data analysis, social signal processing, visual surveillance, intelligent transportation systems and medical imaging. He serves as a reviewer for many international conferences and journals. He is a senior member of the IEEE and a member of the ACM and the IET.

GRANTHAM K.H. PANG obtained his Ph.D. degree from the University of Cambridge in 1986. He was with the Department of Electrical and Computer Engineering, University of Waterloo, Canada, from 1986 to 1996. After that, he joined the Department of Electrical and Electronic Engineering at The University of Hong Kong as an Associate Professor. He has published more than160 technical papers and has authored or co-authored six books. He has also obtained five U.S. patents. His research interests include machine vision for surface defect detection, video surveillance, expert systems for control system design, intelligent control and intelligent transportation systems. Dr. Pang is a Chartered Electrical Engineer, and a member of the IET, HKIE as well as a Senior Member of IEEE. Dr. Pang has acted as consultant to many local and international companies. 